\title{
Chapter 10 \\ LEAP-2017: Comparison of the Type-B Numerical Simulations with Centrifuge Test Results
}

\author{
Majid T. Manzari, Mohamed El Ghoraiby, Mourad Zeghal, \\ Bruce L. Kutter, Pedro Arduino, Andres R. Barrero, Emilio Bilotta, \\ Long Chen, Renren Chen, Anna Chiaradonna, Ahmed Elgamal, \\ Gianluca Fasano, Kiyoshi Fukutake, William Fuentes, Alborz Ghofrani, \\ Stuart K. Haigh, Wen-Yi Hung, Koji Ichii, Dong Soo Kim, \\ Takatoshi Kiriyama, Carlos Lascarro, Gopal S. P. Madabhushi, \\ Vicente Mercado, Jack Montgomery, Mitsu Okamura, Osamu Ozutsumi, \\ Zhijian Qiu, Mahdi Taiebat, Tetsuo Tobita, Thaleia Travasarou, \\ Dimitra Tsiaousi, Kyohei Ueda, Jose Ugalde, Toma Wada, Rui Wang, \\ Ming Yang, Jian-Min Zhang, Yan-Guo Zhou, and Katerina Ziotopoulou
}

\footnotetext{
M. T. Manzari $(\bowtie)$

Department of Civil and Environmental Engineering, George Washington University, Washington, DC, USA

e-mail: manzari@gwu.edu

M. El Ghoraiby

The George Washington University, Washington, DC, USA

M. Zeghal

Department of Civil and Environmental Engineering, Rensselaer Polytechnic Institute, Troy, NY, USA

\section{B. L. Kutter}

Department of Civil and Environmental Engineering, University of California, Davis, CA, USA

P. Arduino · L. Chen · A. Ghofrani

Department of Civil and Environmental Engineering, University of Washington, Seattle, WA, USA
}
A. R. Barrero · M. Taiebat · M. Yang
Department of Civil Engineering, University of British Columbia, Vancouver, BC, Canada
E. Bilotta · A. Chiaradonna · G. Fasano
Department of Civil, Architectural and Environmental Engineering, University of Napoli
Federico II, Naples, Italy
R. Chen $\cdot$ R. Wang $\cdot$ J.-M. Zhang
Department of Hydraulic Engineering, Tsinghua University, Beijing, China
A. Elgamal · Z. Qiu
Department of Structural Engineering, University of California, San Diego, La Jolla, CA, USA 


\begin{abstract}
This paper presents comparisons of 11 sets of Type-B numerical simulations with the results of a selected set of centrifuge tests conducted in the LEAP2017 project. Time histories of accelerations, excess pore water pressures, and lateral displacement of the ground surface are compared to the results of nine centrifuge tests. A number of numerical simulations showed trends similar to those observed in the experiments. While achieving a close match to all measured responses (accelerations, pore pressures, and displacements) is quite challenging, the numerical simulations show promising capabilities that can be further improved with the availability of additional high-quality experimental results.
\end{abstract}

K. Fukutake - T. Kiriyama

Institute of Technology, Shimizu Corporation, Tokyo, Japan

W. Fuentes

Universidad del Norte, Barranquilla, Colombia

S. K. Haigh · G. S. P. Madabhushi

Department of Engineering, Cambridge University, Cambridge, UK

W.-Y. Hung

Department of Civil Engineering, National Central University of Taiwan, Taoyuan City, Taiwan

K. Ichii

Faculty of Societal Safety Science, Kansai University (formerly at Hiroshima University),

Osaka, Japan

D. S. Kim

Department of Civil and Environmental Engineering, Korea Advanced Institute of Science and Technology, Daejeon, South Korea

C. Lascarro · V. Mercado

Department of Civil Engineering, University of British Columbia, Vancouver, BC, Canada

J. Montgomery

Department of Civil Engineering, Auburn University, Auburn, AL, USA

M. Okamura

Department of Civil Engineering, Ehime University, Matsuyama, Japan

O. Ozutsumi

Meisosha Corporation, Tokyo, Japan

T. Tobita

Department of Civil Engineering, Kansai University, Osaka, Japan

T. Travasarou $\cdot$ D. Tsiaousi $\cdot$ J. Ugalde

Fugro, Walnut Creek, LA, USA

K. Ueda

Disaster Prevention Research Institute, Kyoto University, Kyoto, Japan

T. Wada

Department of Civil and Earth Resources Engineering, Kyoto University, Kyoto, Japan

Y.-G. Zhou

Department of Civil Engineering, Zhejiang University, Hangzhou, China

K. Ziotopoulou

Department of Civil and Environmental Engineering, University of California, Davis, CA, USA 


\subsection{Introduction}

LEAP-2017 project is a sequel to the LEAP-2015 project (Kutter et al. 2017; Manzari et al. 2017; Zeghal et al. 2017) that initiated an international collaboration for validation of constitutive and numerical modeling of soil liquefaction and its consequences using high-quality centrifuge tests. The goals of LEAP-2017 are to assess: (1) the repeatability and reproducibility of centrifuge tests, (2) the sensitivity of the experimental results to variation of testing parameters and conditions, and (3) the performance and validity of constitutive models and numerical modeling tools in predicting the observed response. To achieve these goals, the project consisted of a number of steps including a comprehensive numerical simulation exercise. For the LEAP-2017 project, this exercise consisted of three phases: (1) constitutive model calibration, (2) Type-B prediction, and (3) Type-C prediction and sensitivity analyses.

In the first phase, numerical simulation teams used a large number of laboratory tests (El Ghoraiby et al. 2018, 2019) to calibrate their constitutive models. Manzari et al. (2019) provide a summary of the results of the first phase. In the second phase of the project, twelve numerical simulation teams (hereafter referred to as predictors) from different academic institutions and the geotechnical engineering industry across the world participated in the numerical simulation of a selection of the performed centrifuge experiments. These numerical simulations are labeled as "Type-B" since the results of the experiments were unknown to the predictors at the time of their analyses, and only the test configuration and the achieved base excitations and densities were made available to them. As part of this exercise, each predictor team submitted a report discussing key details of the used numerical simulation techniques/platforms. A summary of these reports is also presented in the papers by each predictor team in the proceedings of LEAP-UCD-2017 workshop (Wang et al. 2019; Ozutsumi 2019; Fukutake and Kiriyama 2019; Fasano et al. 2019; Montgomery and Ziotopoulou 2019; Chen et al. 2019; Wada and Ueda 2019; Mercado et al. 2017; Yang et al. 2019; Tsiaousi et al. 2019; Ichii et al. 2019).

This paper presents a summary of key aspects of the Type-B numerical simulations and their comparisons with the experimental data obtained from centrifuge tests (Kutter et al. 2019).

\subsection{LEAP-2017 Centrifuge Experiments}

Similar to the LEAP-2015 project, the LEAP-2017 centrifuge experiments were designed to investigate the lateral spreading of a submerged mildly sloping liquefiable deposit. Figures 10.1 and 10.2 show the baseline schematic of these experiments (Kutter et al. 2017, 2019). The soil specimen is a sloping layer of Ottawa F65 sand with a height of $4 \mathrm{~m}$ (in prototype scale) at the center and a slope of 


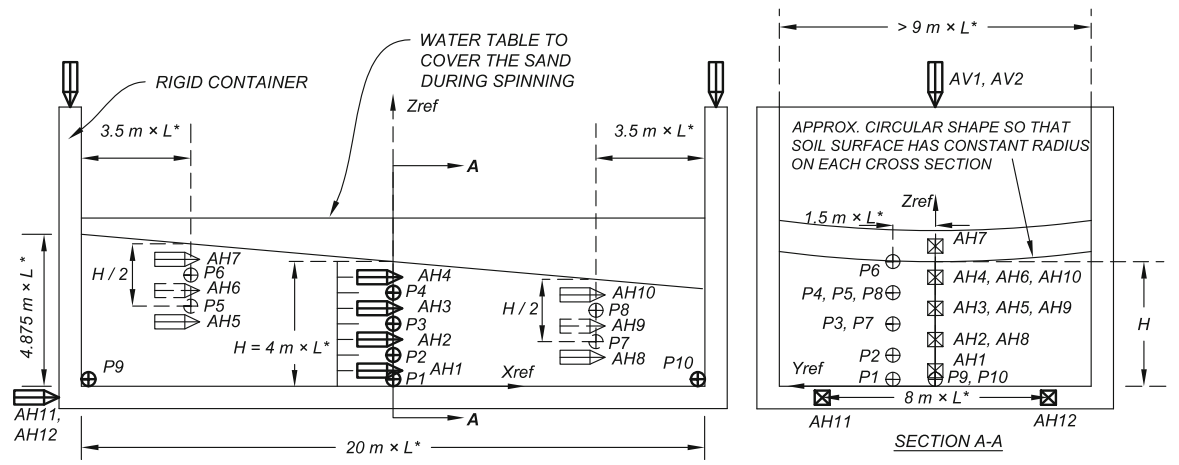

Fig. 10.1 Baseline schematic for the LEAP-2017 experiment for shaking parallel to the axis of the centrifuge (Kutter et al. 2019)
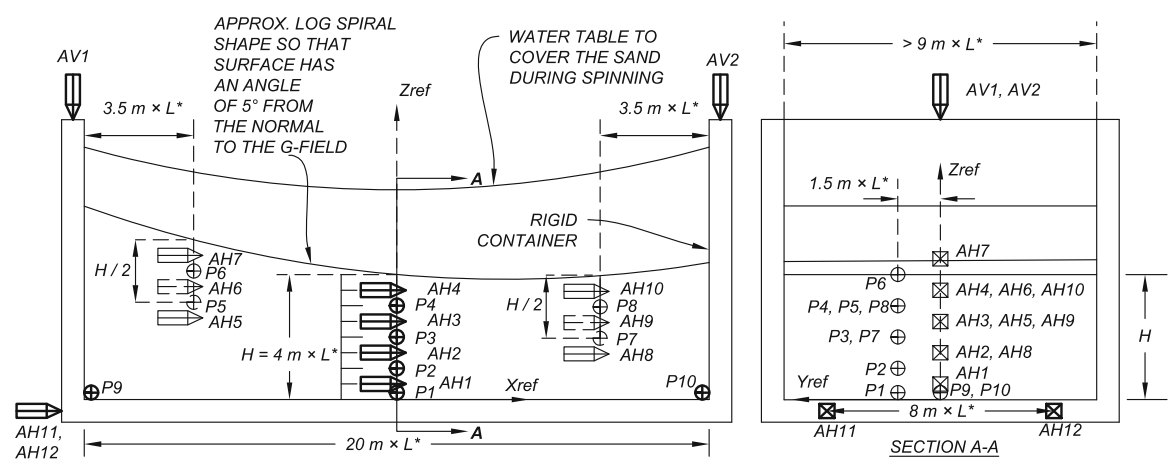

Fig. 10.2 Baseline schematic for the LEAP-2017 experiment for shaking in the circumferential direction of the centrifuge (Kutter et al. 2019)

5 degrees. The prototype soil layer has a length of $20 \mathrm{~m}$ and a width of at least $9 \mathrm{~m}$ (in prototype scale).

The specimen is built in a container with rigid walls. Three arrays of accelerometers and pore pressure transducers are placed in the central section and at $3.5 \mathrm{~m}$ away from the side walls on the right and left of the model. In the transverse direction, the accelerometers are placed in the center while the pore pressure transducers are placed at a distance of $1.5 \mathrm{~m}$ away from the center. In the vertical direction, the sensors were $1.0 \mathrm{~m}$ apart. Tables $10.1 \mathrm{a}$ and $10.1 \mathrm{~b}$ show the specified locations (distance from centerline and the depth) of the pore pressure sensors and accelerometers, respectively, at prototype scale. The actual sensor locations deviated from the specification; the deviation is documented in the papers by individual experimenters or in their data documented in the LEAP-UCD-2017 DesignSafe-CI.

Nine teams of centrifuge modelers from across the world performed 24 centrifuge tests covering a range of achieved densities and shaking intensities. Of these 24 tests, the numerical modelers were asked to simulate nine tests in the Type-B exercise. 
Table 10.1a Specified positions of the pore pressure transducers of LEAP-2017 experiments

\begin{tabular}{l|l|l|l|l|r|r|l|l}
\hline Sensor & P1 & P2 & P3 & P4 & P5 & P6 & P7 & P8 \\
\hline x-pos. (m) & 0.0 & 0.0 & 0.0 & 0.0 & -6.5 & -6.5 & 6.5 & 6.5 \\
\hline Depth (m) & 4.0 & 3.0 & 2.0 & 1.0 & 2.0 & 1.0 & 2.0 & 1.0 \\
\hline
\end{tabular}

Table 10.1b Specified positions of the accelerometers of LEAP-2017 experiments

\begin{tabular}{l|l|l|l|l|r|r|r|l|l|l}
\hline Sensor & AH1 & AH2 & AH3 & AH4 & AH5 & AH6 & AH7 & AH8 & AH9 & AH10 \\
\hline x-pos. (m) & 0.0 & 0.0 & 0.0 & 0.0 & -6.5 & -6.5 & -6.5 & 6.5 & 6.5 & 6.5 \\
\hline Depth (m) & 3.5 & 2.5 & 1.5 & 0.5 & 2.5 & 1.5 & 0.5 & 2.5 & 1.5 & 0.5 \\
\hline
\end{tabular}

Table 10.2 Summary of centrifuge experiments selected for LEAP-2017 Type-B simulations

\begin{tabular}{l|l|l|l|l|l|l}
\hline $\begin{array}{l}\text { Centrifuge } \\
\text { test }\end{array}$ & $\mathrm{g} *$ & $\begin{array}{l}\text { Shaking } \\
\text { direction }\end{array}$ & $\begin{array}{l}\text { Radius } \\
\text { of centrifuge } \\
(\mathrm{m})\end{array}$ & $\begin{array}{l}\mu * l \\
\mathrm{~g} * \\
\mathrm{cSt})\end{array}$ & $\begin{array}{l}\text { Soil } \\
\text { density } \\
\left(\mathrm{kg} / \mathrm{m}^{3}\right)\end{array}$ & $D_{\mathrm{r}}(\%)$ \\
\hline CU-2 & 40 & Tangential & 3.56 & 1.175 & 1605.8 & 46.3 \\
\hline Ehime-2 & 40 & Axial & 1.184 & 1.0 & 1656.55 & 64.4 \\
\hline KAIST-1 & 40 & Axial & 5.00 & 0.897 & 1701.2 & 79.4 \\
\hline KAIST-2 & 40 & Axial & 5.00 & 0.936 & 1592.5 & 41.4 \\
\hline KyU-3 & 44.4 & Tangential & 2.5 & 0.991 & 1637 & 57.6 \\
\hline NCU-3 & 26 & Axial & 2.716 & - & 1652 & 62.8 \\
\hline UCD-1 & 43 & Tangential & 1.094 & 1.0 & 1665 & 67.3 \\
\hline UCD-3 & 43 & Tangential & 1.094 & 1.0 & 1658 & 64.9 \\
\hline ZJU-2 & 30 & Axial & 4.315 & 1.0 & 1606 & 46.4 \\
\hline
\end{tabular}

* Centrifugal acceleration

Table 10.2 shows a summary of the experiments selected for the LEAP-2017 Type-B simulations. The table lists the main characteristics of each experiment including: centrifugal acceleration scale $\mathrm{g}^{*}$ with respect to gravity $1 \mathrm{~g}$, shaking direction relative to centrifugal axis, radius of centrifuge, viscosity of the pore fluid, and the reported achieved soil density.

\subsection{Type-B Numerical Simulations}

Following submission of the calibration simulations (phase 1), the recorded base motions along with the achieved geometry and density of the test specimens for the selected centrifuge tests were provided to all the predictors on July 28, 2017. The predictors were asked to submit their simulation of the nine centrifuge tests by September 30, 2017. The information provided for the majority of the selected tests included in-flight CPT measurements just before the first destructive base motion. Table 10.3 lists the numerical prediction teams (predictors) who participated in the Type-B simulation exercise. 
Table 10.3 Numerical simulation teams

\begin{tabular}{|c|c|c|c|}
\hline No & Numerical simulation team & Constitutive model & $\begin{array}{l}\text { Analysis } \\
\text { platform }\end{array}$ \\
\hline 1 & Tsinghua University & Tsinghua constitutive model & OpenSees \\
\hline 2 & Meisosha Corporation & Cocktail glass model & FLIP Rose \\
\hline 3 & Shimizu Corporation & Bowl model & HiPER \\
\hline 4 & University of Napoli Federico II & Hypoplastic model & Plaxis \\
\hline 5 & UC Davis-Auburn University & PM4Sand model & FLAC-2D \\
\hline 6 & University of Washington & $\begin{array}{l}\text { Manzari-Dafalias model/PM4Sand } \\
\text { model }\end{array}$ & OpenSees \\
\hline 7 & Kyoto University & Cocktail glass model & FLIP TULIP \\
\hline 8 & Universidad del Norte & ISA-hypoplasticity model & ABAQUS \\
\hline 9 & University of British Columbia & SANISand & FLAC-3D \\
\hline 10 & $\begin{array}{l}\text { University of California, San } \\
\text { Diego }\end{array}$ & PDMY & OpenSees \\
\hline 11 & Fugro West & PM04 model/UBCSAND & FLAC-2D \\
\hline 12 & Hiroshima (Kansai) University & Cocktail glass model & FLIP Rose \\
\hline
\end{tabular}

The constitutive model and the finite element/difference platform used by each numerical simulation team are also shown in the following Table 10.3. It is noted that the fifth team in the above list (UC Davis-Auburn University Team) submitted four different predictions for each of the nine centrifuge tests. The basis of this selection was the variation between the relative densities achieved at the different facilities and between those and the target relative density. The differences between these simulations are discussed in details in by Montgomery and Ziotopoulou (2019). These simulations are labeled as $5 \mathrm{a}$ to $5 \mathrm{~d}$ in this paper, with simulations $5 \mathrm{a}$ and $5 \mathrm{~b}$ representing the team's "best estimate." The sixth team in the above list (University of Washington) used two different models (DM04 and PM4Sand) in their simulations which are identified as $6 \mathrm{a}$ and $6 \mathrm{~b}$ in the following discussions. Similarly, the 11th numerical simulation team (Fugro-West team) used two different constitutive models (PM4Sand and UBC Sand) in their simulations of three of the nine centrifuge tests. These will be labeled, respectively, as 11a and $11 \mathrm{~b}$ simulations in the following discussions.

The numerical simulation teams were requested to submit a Type-B simulation report discussing the steps followed in the prediction of the centrifuge experiments. The Type-B prediction report discussed the main features of the numerical analysis platform used in the simulation, the model geometry and the discretization details, the boundary conditions of the numerical model, the solution algorithm employed, and the assumptions used in the analyses. More detailed information about each constitutive model and the numerical simulation techniques used by each team are provided in separate papers and reports (Wang et al. 2019; Ozutsumi 2019; Fukutake and Kiriyama 2019; Fasano et al. 2019; Montgomery and Ziotopoulou 2019; Chen et al. 2019; Wada and Ueda 2019; Mercado et al. 2017; Yang et al. 2019; Tsiaousi et al. 2019; Ichii et al. 2019). The team from Hiroshima University submitted only one simulation for the Kyoto test which is described in details in Ichii et al. (2019) and will not be discussed herein. 


\subsection{Summary of Type-B Simulations Results}

The results of the Type-B numerical simulations submitted by the 11 simulation teams for the selected LEAP-2017 centrifuge tests consist of 93 simulation sets, each containing time histories of predicted accelerations, excess pore water pressures, and displacements at selected locations within the centrifuge specimens. Due to space limitation, only a small subset of this data is presented herein. In the following sections, selected time histories of excess pore pressure, spectral accelerations, and lateral displacements are presented and discussed. While the data presented and analyzed here does not cover the entire dataset, the main objective is to provide representative samples of the performance of each simulation in comparison with the experimental data.

\subsubsection{Excess Pore Water Pressure Time Histories}

Figures $10.3,10.4,10.5,10.6,10.7,10.8$, and 10.9 show detailed comparisons of the predicted time histories of excess pore water pressures computed at the central section of the centrifuge specimen (pore pressure sensors 1 to 4 ) with the results

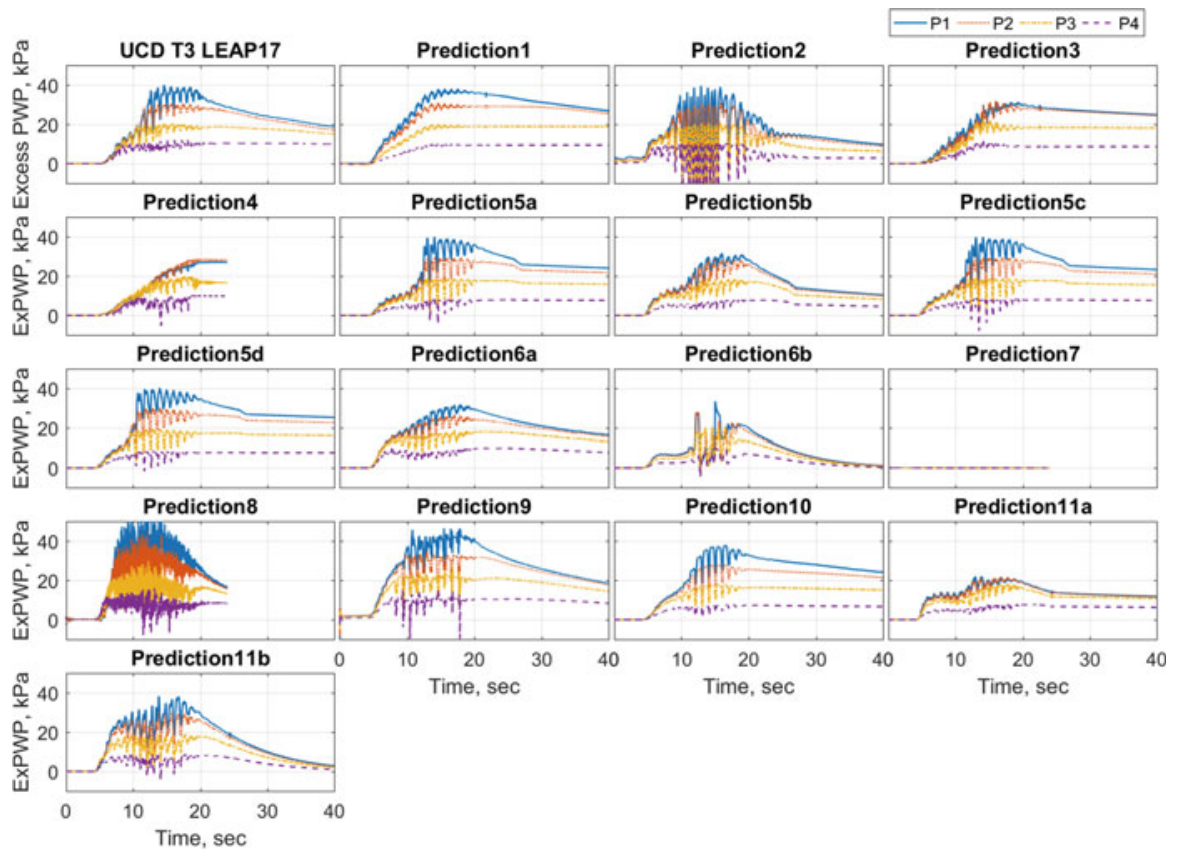

Fig. 10.3 Comparison of the predicted time histories of excess pore water pressures computed at the central section of the soil specimen for UCD-3 test 


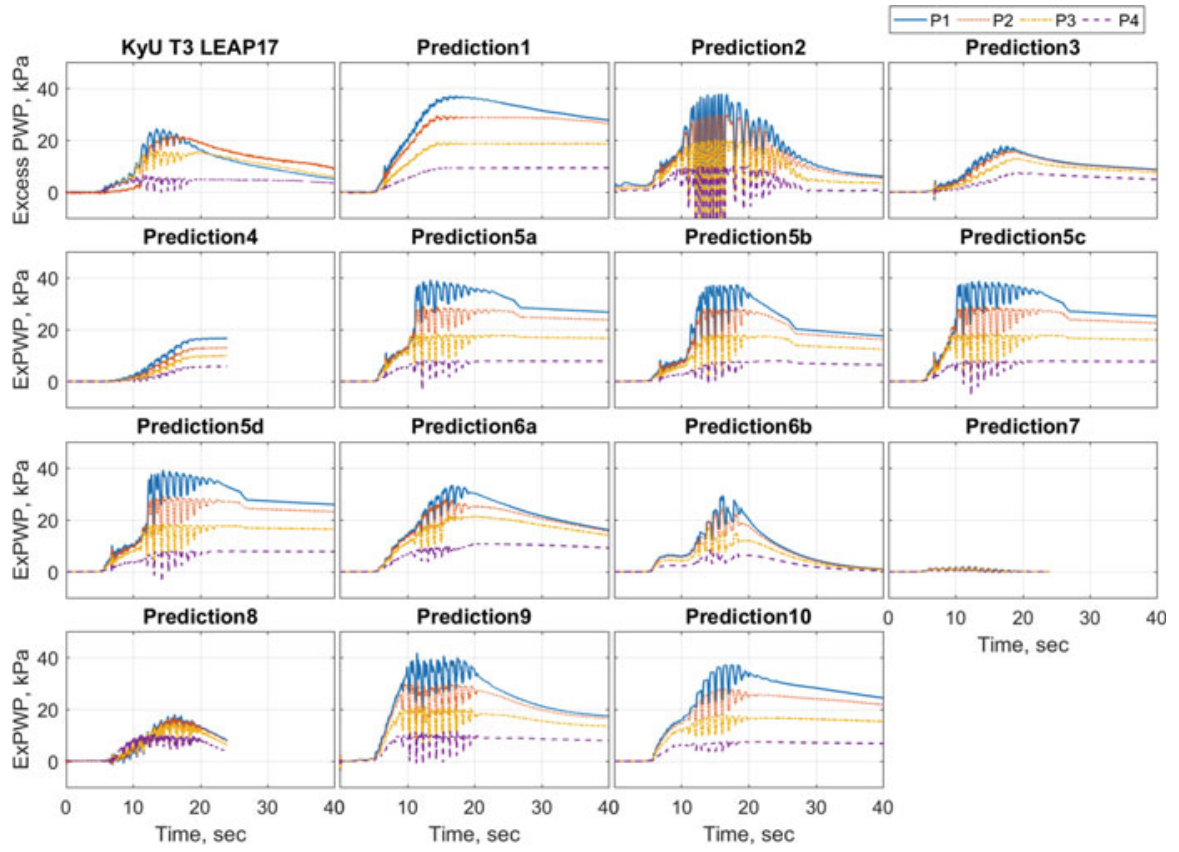

Fig. 10.4 Comparison of the predicted time histories of excess pore water pressures computed at the central section of the soil specimen for KyU-3 test

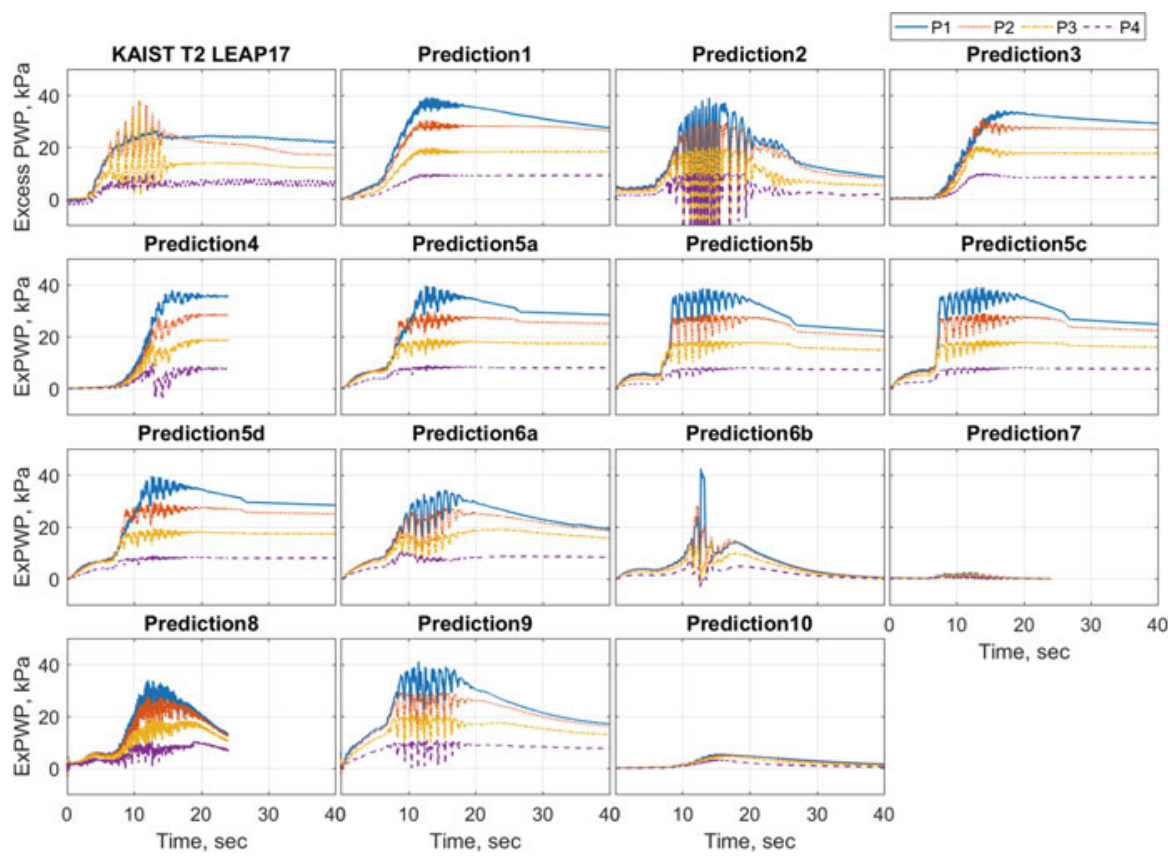

Fig. 10.5 Comparison of the predicted time histories of excess pore water pressures computed at the central section of the soil specimen for KAIST-2 test 


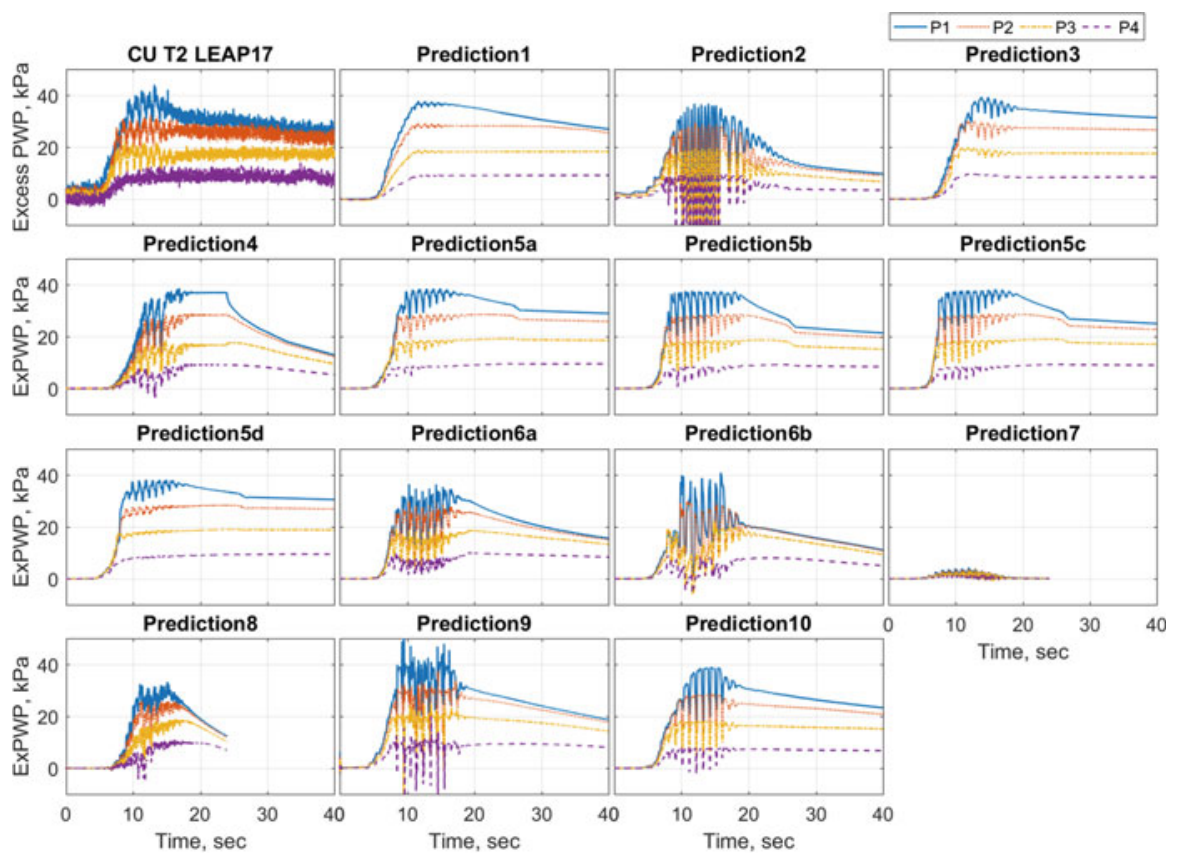

Fig. 10.6 Comparison of the predicted time histories of excess pore water pressures computed at the central section of the soil specimen for CU-2 test

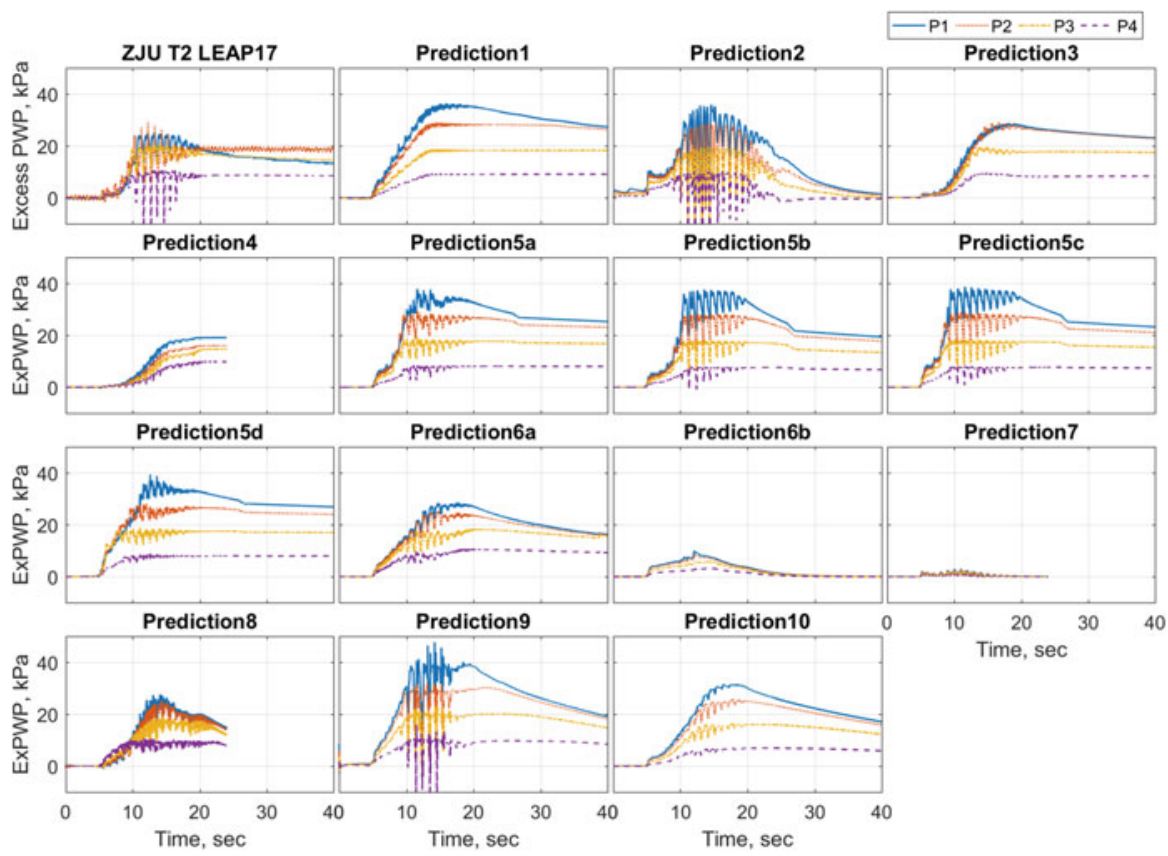

Fig. 10.7 Comparison of the predicted time histories of excess pore water pressures computed at the central section of the soil specimen for ZJU-2 test 


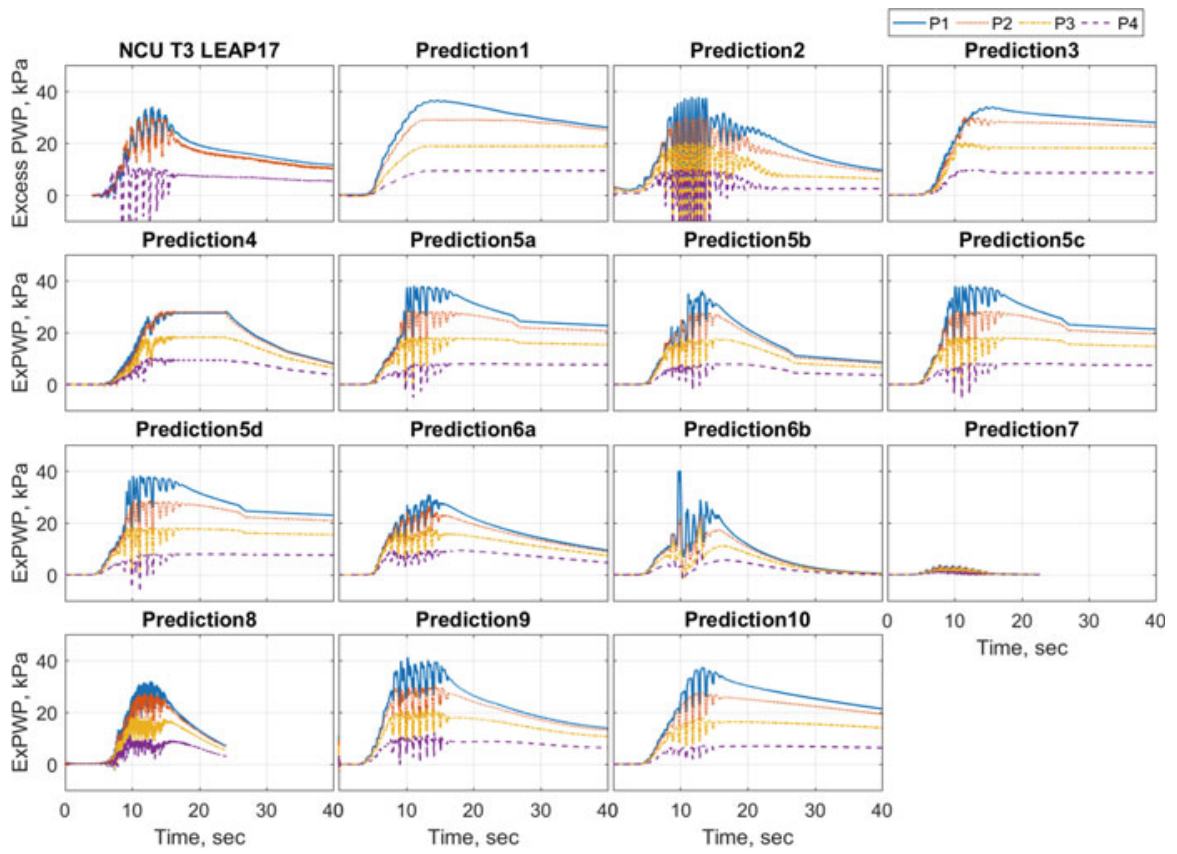

Fig. 10.8 Comparison of the predicted time histories of excess pore water pressures computed at the central section of the soil specimen for NCU-3 (bottom) test

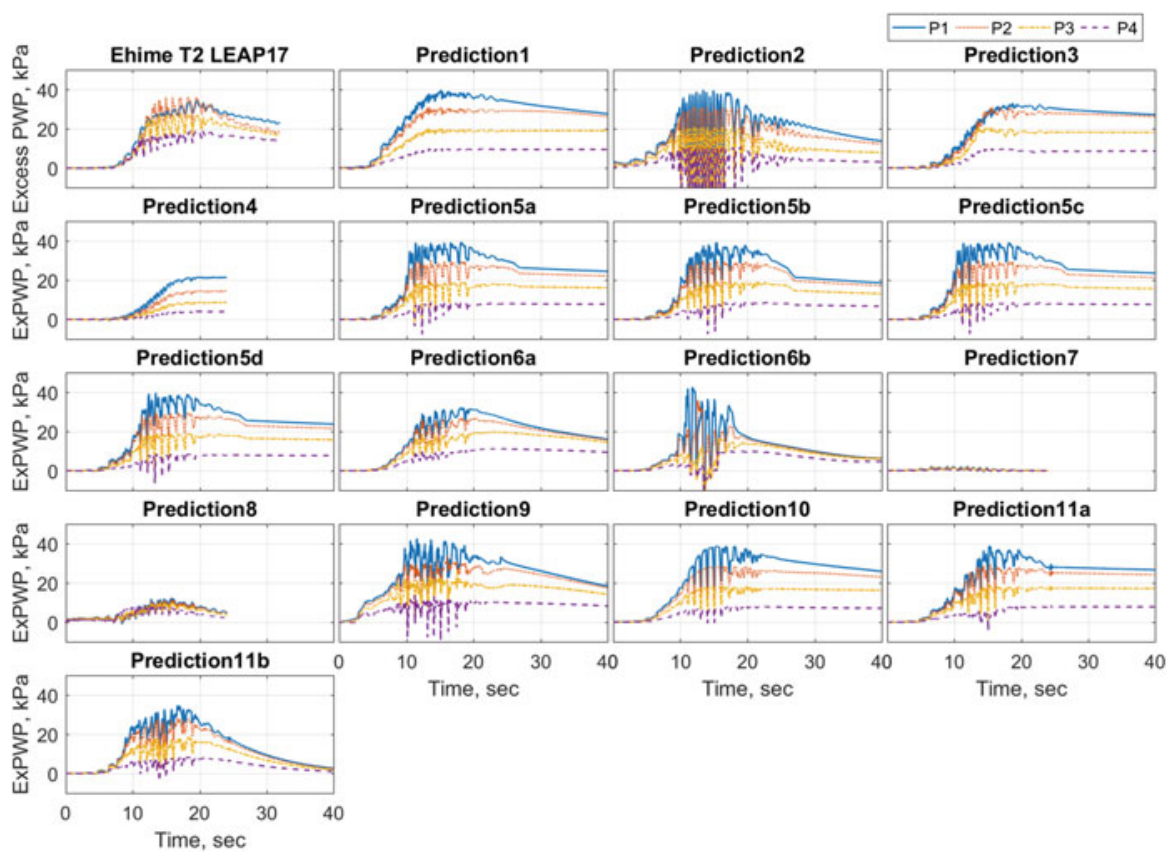

Fig. 10.9 Comparison of the predicted time histories of excess pore water pressures computed at the central section of the soil specimen for Ehime-2 test 
of a few selected centrifuge tests conducted at UC Davis (UCD T3, Carey et al. 2019), Kyoto University (KyU-3, Vargas Tapia et al. 2019), KAIST (KAIST-2, Kim et al. 2019), Cambridge University (CU-2, Madabhushi et al. 2019), Zhejiang University (ZJU-2, Liu et al. 2019), National Central University of Taiwan (NCU-3, Hung and Liao 2019), and Ehime (Ehime-2, Okamura and Nurani Sjafruddin 2019) as part of LEAP-2017 project (Kutter et al. 2019). Qualitative similarities with the experimental curves are noted for the majority of the predictions, except for Prediction 7 in which a calibration error, later noticed by the predictors, produced significantly underpredicted excess pore pressures.

A close examination of the pore pressures time histories illustrated in Figs. 10.3, $10.4,10.5,10.6,10.7,10.8$, and 10.9 shows the following trends:

- Except for the simulation of the CU-2 test, Prediction 1 shows a larger maximum excess pore water pressure compared to the experimentally observed values with a relatively faster buildup and slower dissipation.

- Compared to the experimental results, Prediction 2 shows more dilative responses with larger oscillations when the excess pore pressures reach their maximum values. Moreover in a few cases (e.g., KyU-3), the maximum excess pore water pressures are overpredicted. The predicted pore water pressure time histories show faster dissipation than the experimentally observed time histories.

- In most cases, Prediction 3 shows trends that are reasonably close to the observed experimental responses, but in a few cases, the maximum excess pore pressures are slightly underpredicted.

- Prediction 4 only shows the computed responses up to the end of shaking and do not include the dissipation phase, except for the case of NCU-3. In few cases (e.g., UCD-3 test), the maximum excess pore water pressures are underpredicted.

- Predictions 5a, 5c, and 5d show similar buildup and dissipation trends and are different from Prediction $5 \mathrm{~b}$ in the dissipation phase of the pore pressure time histories. The maximum pore pressures in these predictions are in reasonable agreement with the experimentally observed values in the majority of cases except for two cases where they are visibly underpredicted (UCD-3) or overpredicted (KyU-3) in Prediction 5b.

- Prediction 6a shows trends comparable to experimentally observed pore pressure time histories. However, the maximum excess pore pressures are visibly underpredicted in the case of UCD-3 and they are overpredicted in the cases of KyU-3.

- Prediction $6 \mathrm{~b}$ shows some large spikes in the pore pressure time histories. These spikes might be related to numerical implementation of the constitutive model rather than the model performance. These predictions underpredict the observed excess pore water pressure time histories in the majority of the simulated tests.

- Prediction 7 significantly underpredicts the excess pore pressure time histories. This is likely due to a systematic error in application of the method used in the simulations.

- Prediction 8 shows a faster dissipation than that observed in the experiments in all the predicted time histories. The maximum excess pore pressures are reasonably 
well predicted in a few cases and are underpredicted in the case of $\mathrm{KyU}-3, \mathrm{CU}-2$, and Ehime-2.

- Prediction 9 shows trends that are comparable to experimentally observed pore pressure time histories. However, the maximum excess pore pressures are overpredicted in the cases of KyU-3 and ZJU-2.

- Prediction 10 shows comparable trends with the experimental data. In most cases, a slower dissipation phase is predicted. The maximum excess pore pressures are overpredicted in the cases of KyU-3.

- Prediction 11a is in good agreement with the measured responses in the case of Ehime-2 and underpredicts the maximum excess pore pressures in the case of UCD-3. Predictions were not submitted for the other experiments.

- Prediction $11 \mathrm{~b}$ shows comparable trends with the experimental data obtained in the case of UCD-3 and Ehime-2. Slightly faster dissipation rates are predicted compared to the experimentally observed rates. Predictions were not submitted for the other experiments.

\subsubsection{Acceleration Time Histories and Spectral Accelerations}

Figures $10.10,10.11,10.12,10.13,10.14,10.15$, and 10.16 show examples of acceleration time histories predicted by the different simulation teams and compare them with the time histories of accelerations obtained from the centrifuge test results. The following observations are noted:
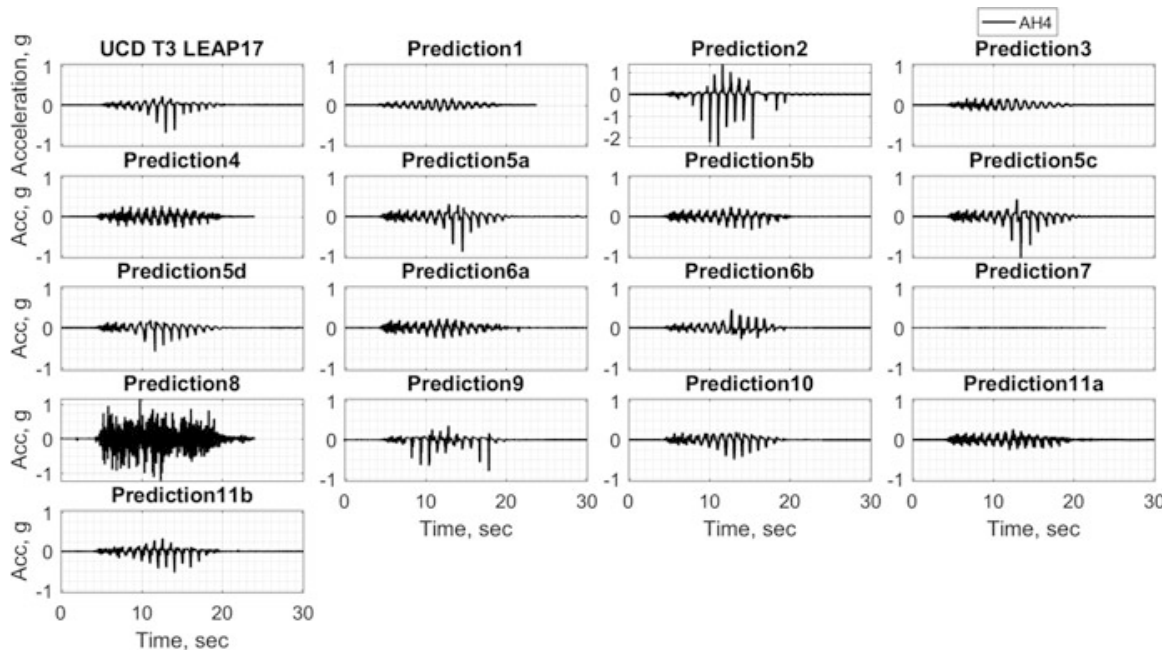

Fig. 10.10 Comparison of the acceleration time histories computed at the location of AH4 in the central section of the soil specimen for UCD-3 test 


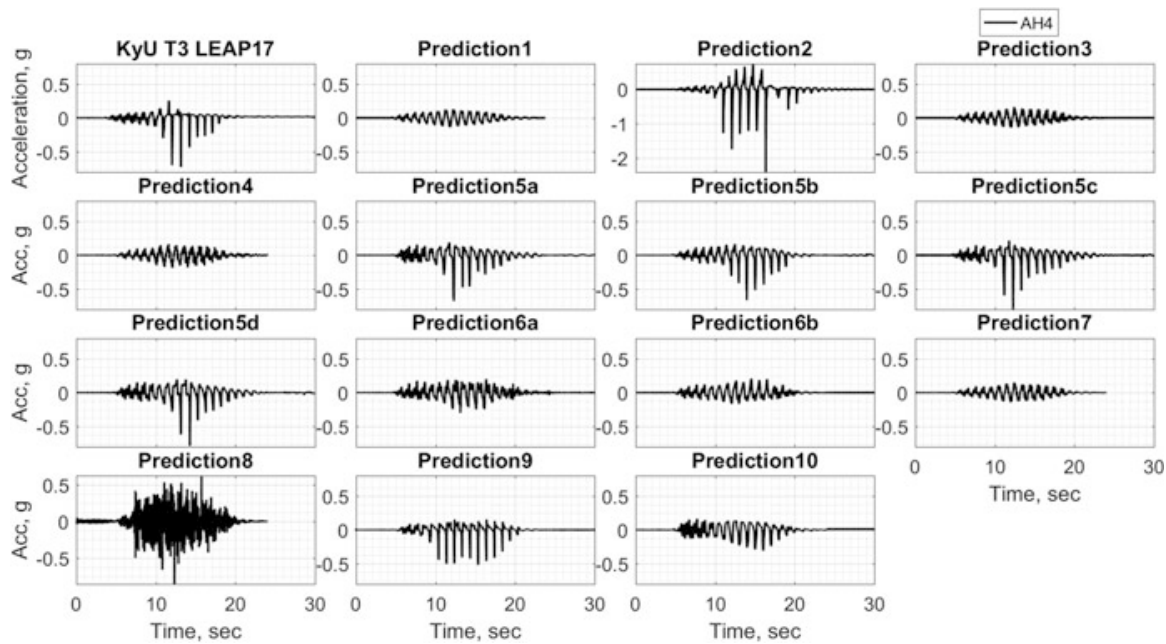

Fig. 10.11 Comparison of the acceleration time histories computed at the location of AH4 in the central section of the soil specimen for KyU-3 test
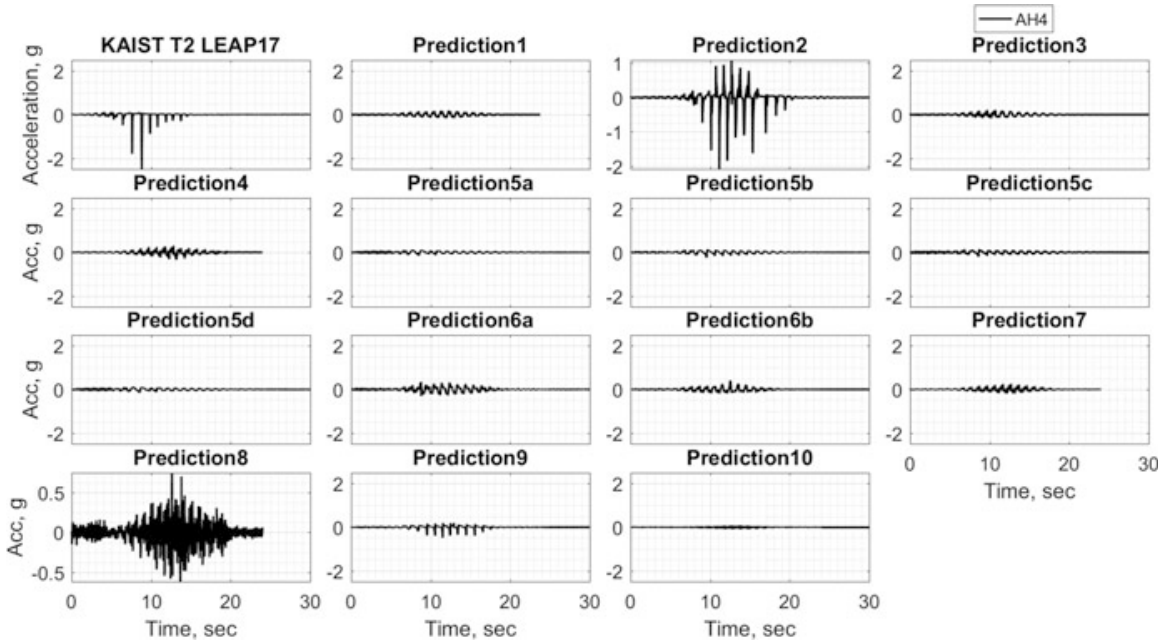

Fig. 10.12 Comparison of the acceleration time histories computed at the location of AH4 in the central section of the soil specimen for KAIST-2 test

- The experimental data of UCD-3 test show that the acceleration time history at AH4 (near the ground surface) is marked by dilation spikes (Fig. 10.10). This feature has been captured in a few predictions (Predictions 2, 5a, 5c, 5d, 6b, 9, 10, $11 \mathrm{~b})$ with varying degrees of success. 


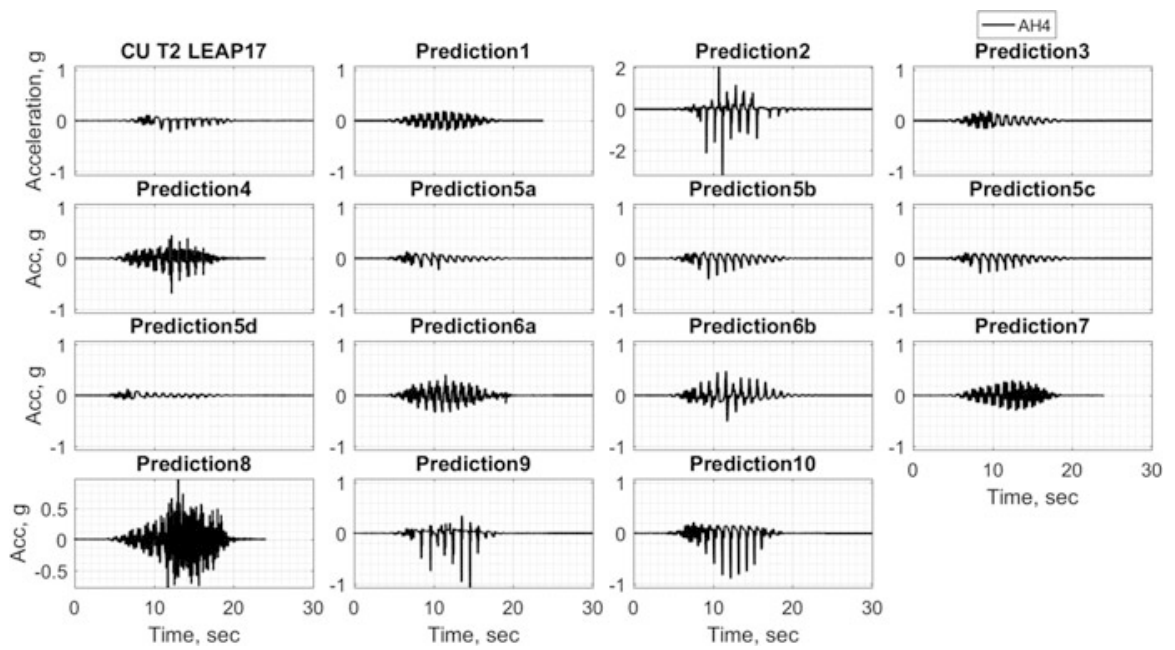

Fig. 10.13 Comparison of the acceleration time histories computed at the location of AH4 in the central section of the soil specimen for CU-2 test

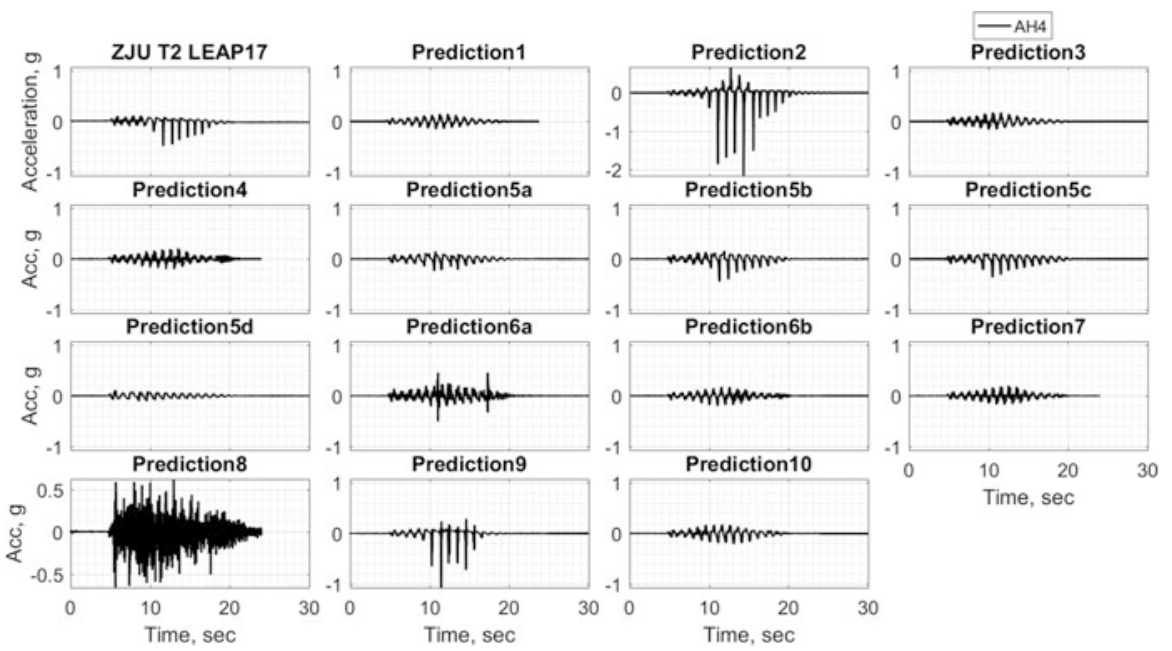

Fig. 10.14 Comparison of the acceleration time histories computed at the location of AH4 in the central section of the soil specimen for ZJU-2 test

- Similar dilation spikes are also present in the AH4 acceleration time histories reported in KyU-3 test (Fig. 10.11). Again, a few predictions show similar dilation spikes (Predictions 2, 5a, 5b, 5c, 5d, 9, 10). Smaller dilation spikes appear in Predictions $6 \mathrm{a}$ and $6 \mathrm{~b}$ as well. 


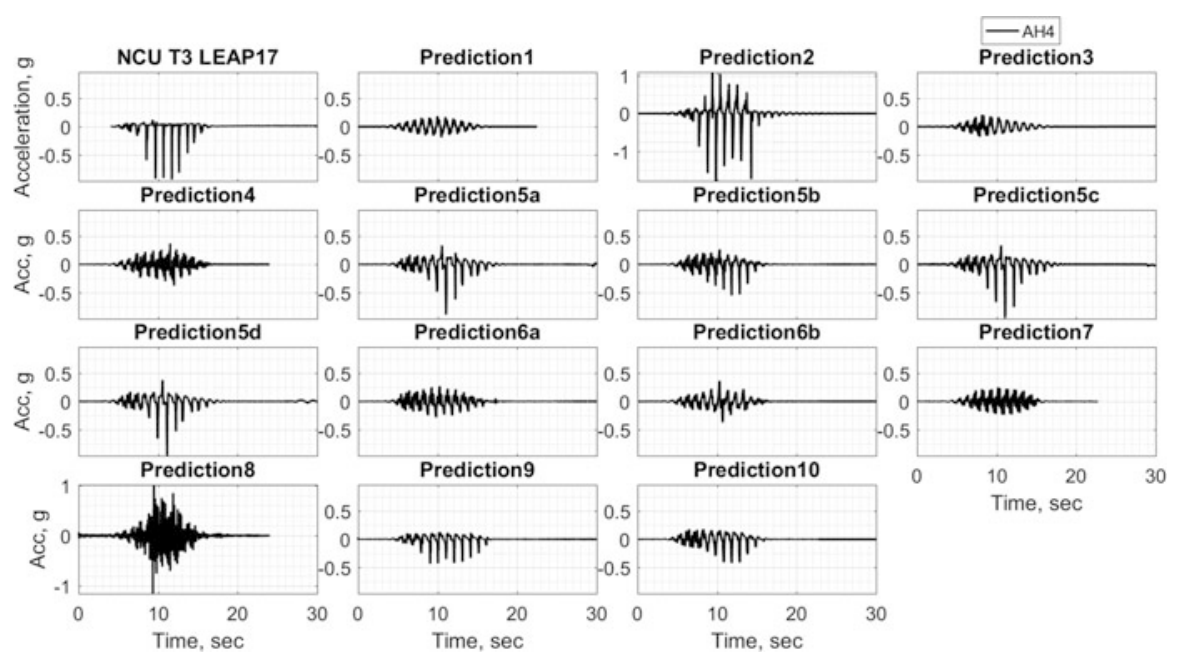

Fig. 10.15 Comparison of the acceleration time histories computed at the location of AH4 in the central section of the soil specimen for NCU-3

- The dilation spikes observed in the CU-2 and ZJU-2 tests (Figs. 10.13 and 10.14) are also present in Predictions 2, 5a, 5b, 5c, 6a, 6b, 7, 9, and 10.

- The measured accelerations at AH4 in the KAIST-2 (Fig. 10.12) and NCU-3 (Fig. 10.15) tests show much stronger dilation spikes compared to other tests. These large spikes are well captured in Predictions 5 a, 5c, and 5d. Predictions 2, 9, and 10 also show large spikes in the acceleration time histories computed at AH4.

- The acceleration time history recorded at AH4 in the Ehime-2 test (Fig. 10.16) shows somewhat smaller dilation spikes (compared to those in the KAIST-2 and NCU-3 tests) in the positive direction. Again a few predictions (5a, 5b, 5c, 5d, $9,10,11 \mathrm{a}, 11 \mathrm{~b})$ show dilation spikes of similar magnitude.

The spectral accelerations shown in Figs. 10.17, 10.18, 10.19, 10.20, 10.21, 10.22 , and 10.23 show details of high-frequency contents in each of the acceleration time histories discussed above. In these figures, Sa1, Sa2, Sa3, and $\mathrm{Sa} 4$ stand for spectral accelerations corresponding to accelerations A1, A2, A3, and A4, respectively. It is interesting to note that a number of predictions are able to capture the $1 \mathrm{~Hz}$ component of the accelerations at different depths quite well. Note also, that a few predictions have higher-frequency contents similar to the observed accelerations and a few other have damped out the high-frequency harmonics. These aspects of the numerical simulations are attributed less to the performance of the constitutive model and more to the numerical damping and other damping mechanisms (e.g., Rayleigh damping) introduced in the numerical simulations. 


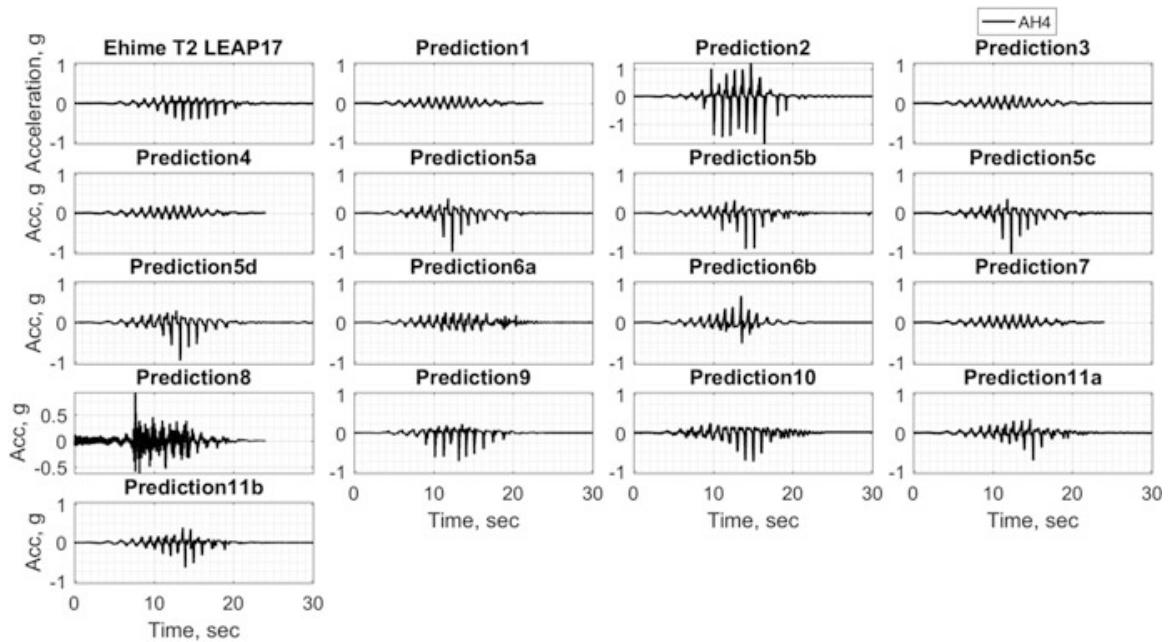

Fig. 10.16 Comparison of the acceleration time histories computed at the location of AH4 in the central section of the soil specimen for Ehime-2 test

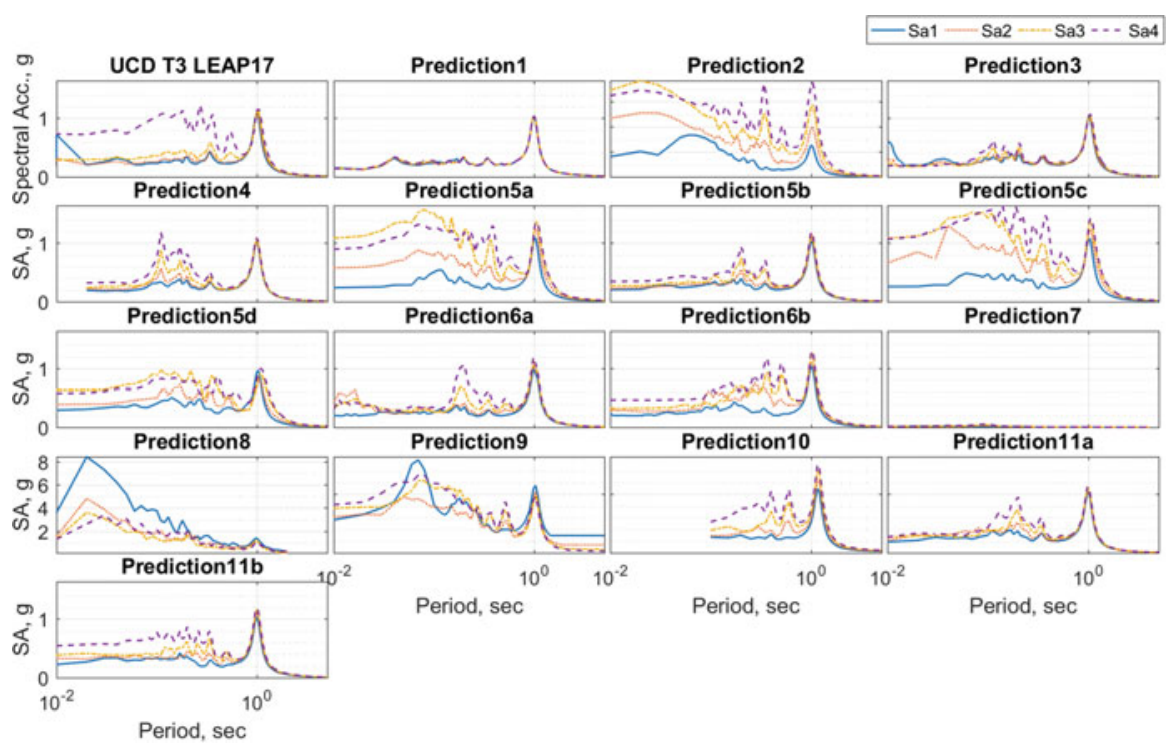

Fig. 10.17 Comparison of the spectral acceleration computed at the central section of the soil specimen for UCD-3 test 


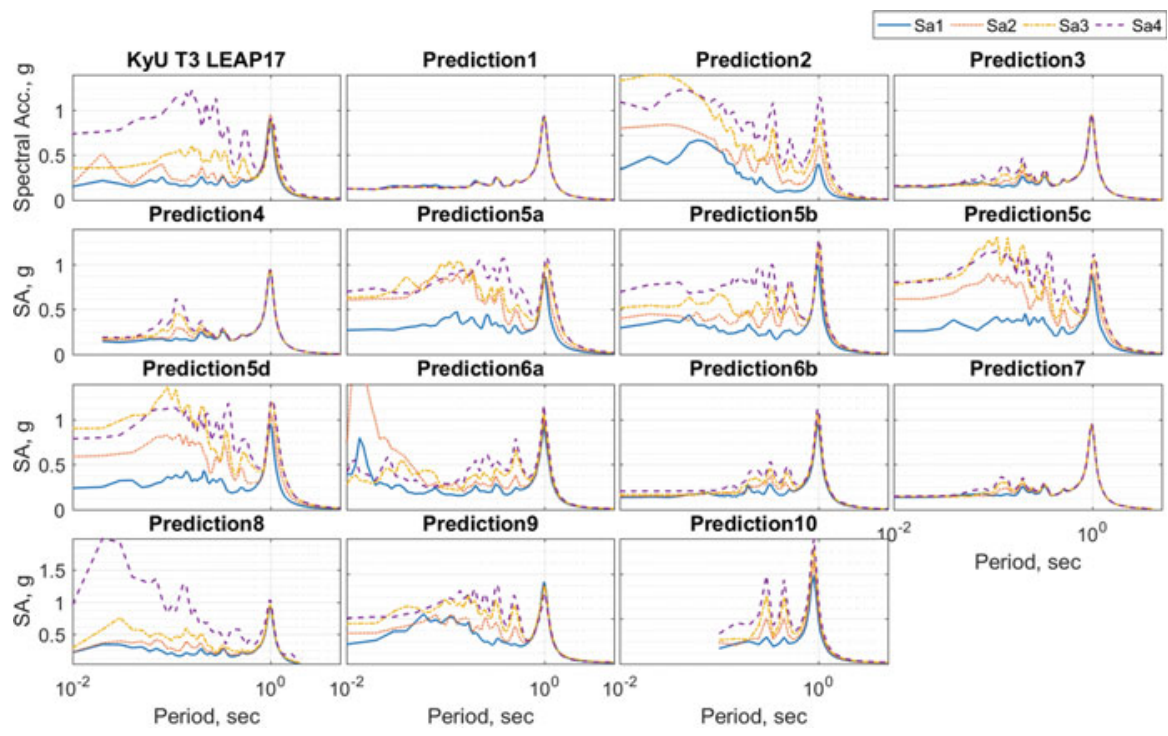

Fig. 10.18 Comparison of the spectral acceleration computed at the central section of the soil specimen for KyU-3 test

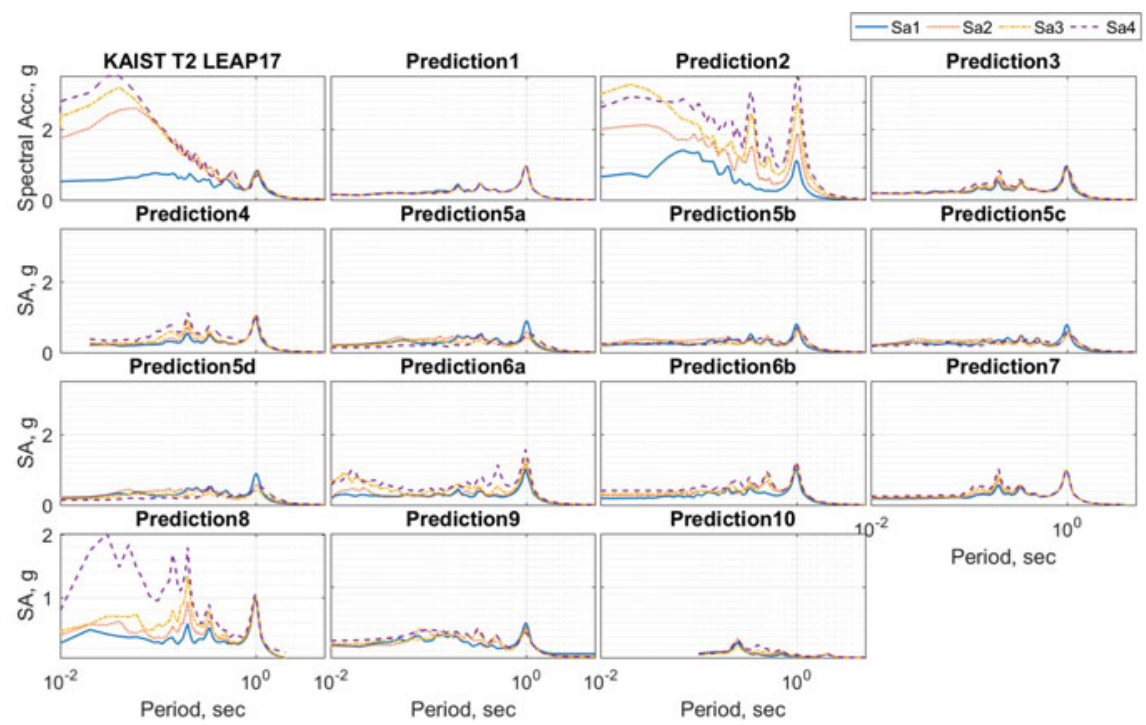

Fig. 10.19 Comparison of the spectral acceleration computed at the central section of the soil specimen for KAIST-2 test 


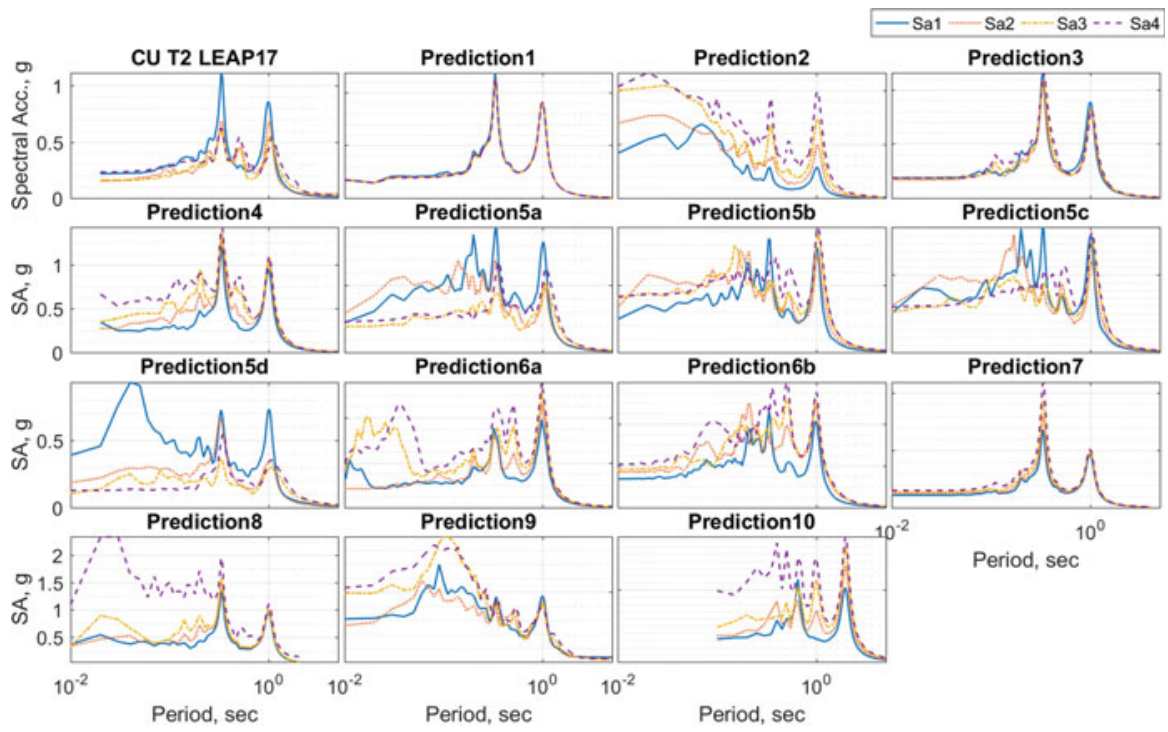

Fig. 10.20 Comparison of the spectral acceleration computed at the central section of the soil specimen for CU-2 test

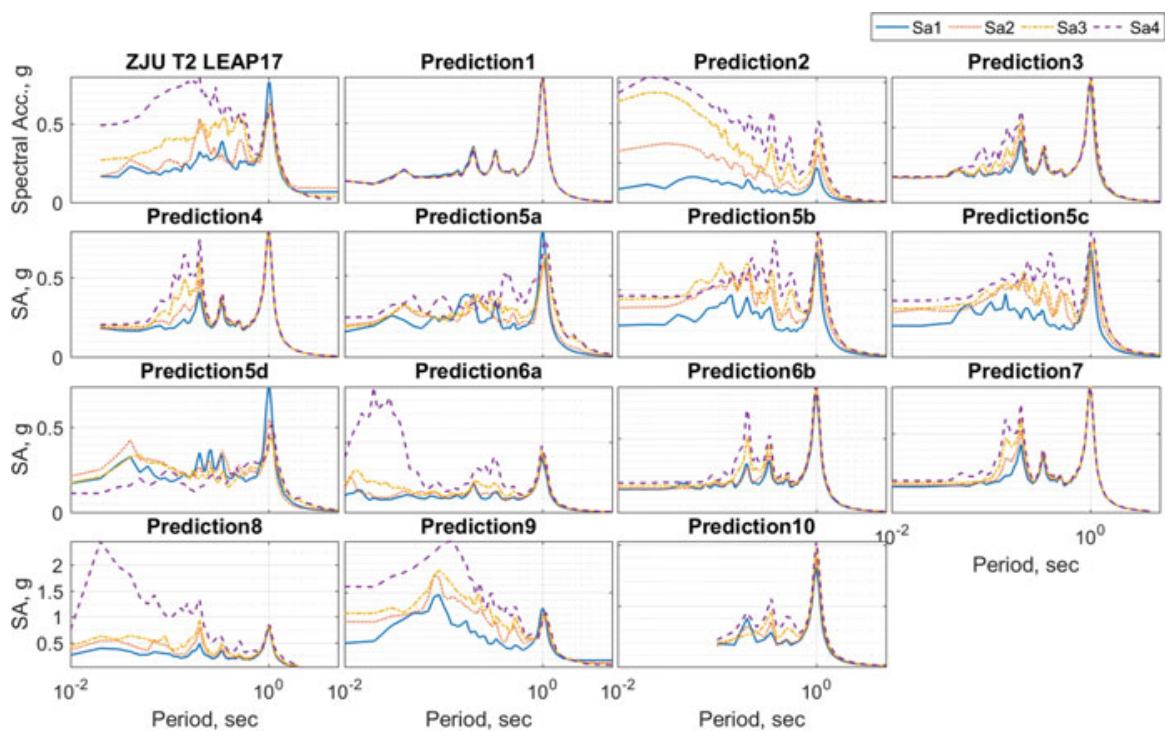

Fig. 10.21 Comparison of the spectral acceleration computed at the central section of the soil specimen for ZJU-2 test 


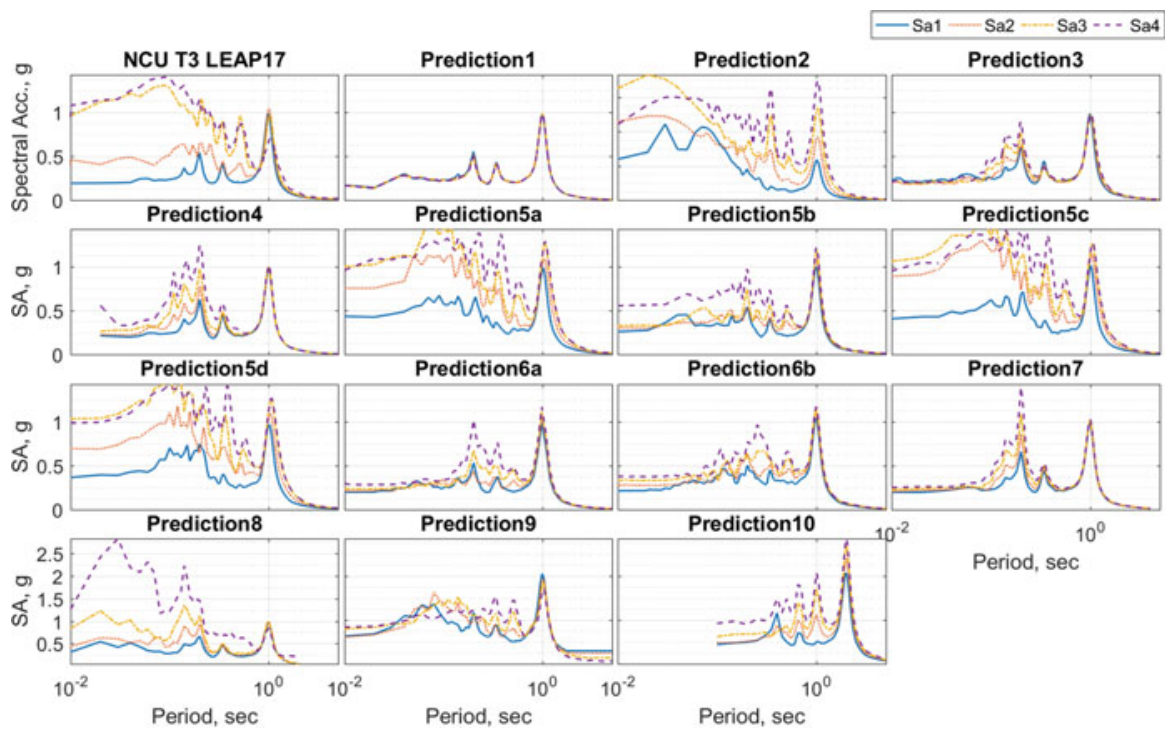

Fig. 10.22 Comparison of the spectral acceleration computed at the central section of the soil specimen for NCU-3 test

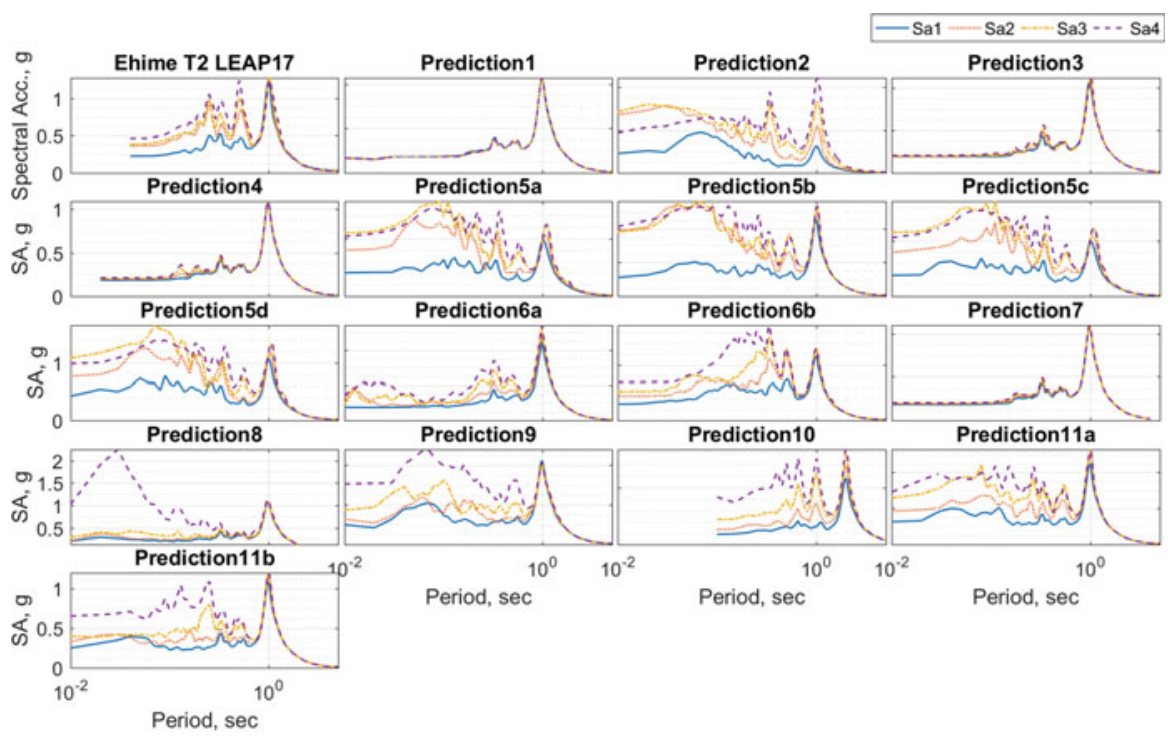

Fig. 10.23 Comparison of the spectral acceleration computed at the central section of the soil specimen for Ehime-2 test 


\subsubsection{Lateral Displacements}

The lateral displacements at the surface of the centrifuge specimens were measured by tracking the locations of surface markers using the photos taken by high-speed cameras as described in Kutter et al. (2019). In two (UCD-1 and UCD-3) out of the nine centrifuge tests selected for Type-B numerical simulations, the data obtained from high-speed cameras were used to construct a time history of surface displacement in the middle portion of the slope. In five other tests (CU-2, Ehime 2, KAIST-2, NCU-3, ZJU-3), the final locations of the surface markers measured after the end of the test and time histories of dynamic displacements computed from measured acceleration time histories were used to reconstruct surface displacement time histories (Kutter et al. 2019). In the case of the KAIST-1 and KyU-3 tests, surface marker data was not available or sufficiently consistent to reconstruct a time history of the surface displacements. In these cases, only dynamic components of the surface displacements were computed by double integration of the difference between the accelerations recorded at AH1 and AH4 (Kutter et al. 2019). Hence, it is important to note that in the following comparisons of the simulated time histories with the results of centrifuge tests except for UCD-1 and UCD-3 tests, the rest of the "experimental" results are computed surface displacement time histories obtained from the final locations of surface markers and double integration of the measured acceleration time histories.

Based on the results illustrated in Figs. 10.24, 10.25, 10.26, 10.27, 10.28, 10.29, 10.30, and 10.31, the following observations can be made:
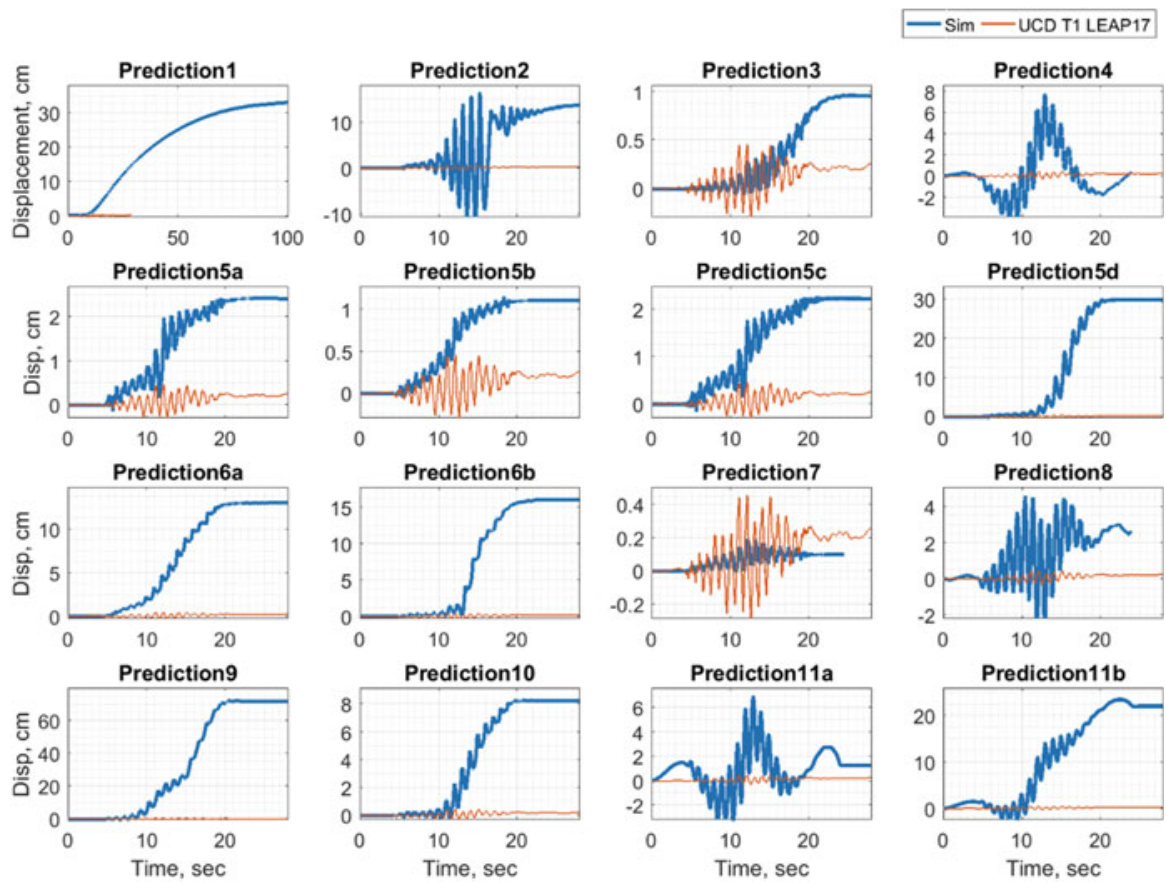

Fig. 10.24 Comparisons of the numerical simulations of lateral displacement with the measured time history of surface displacement in the UCD-1 test 

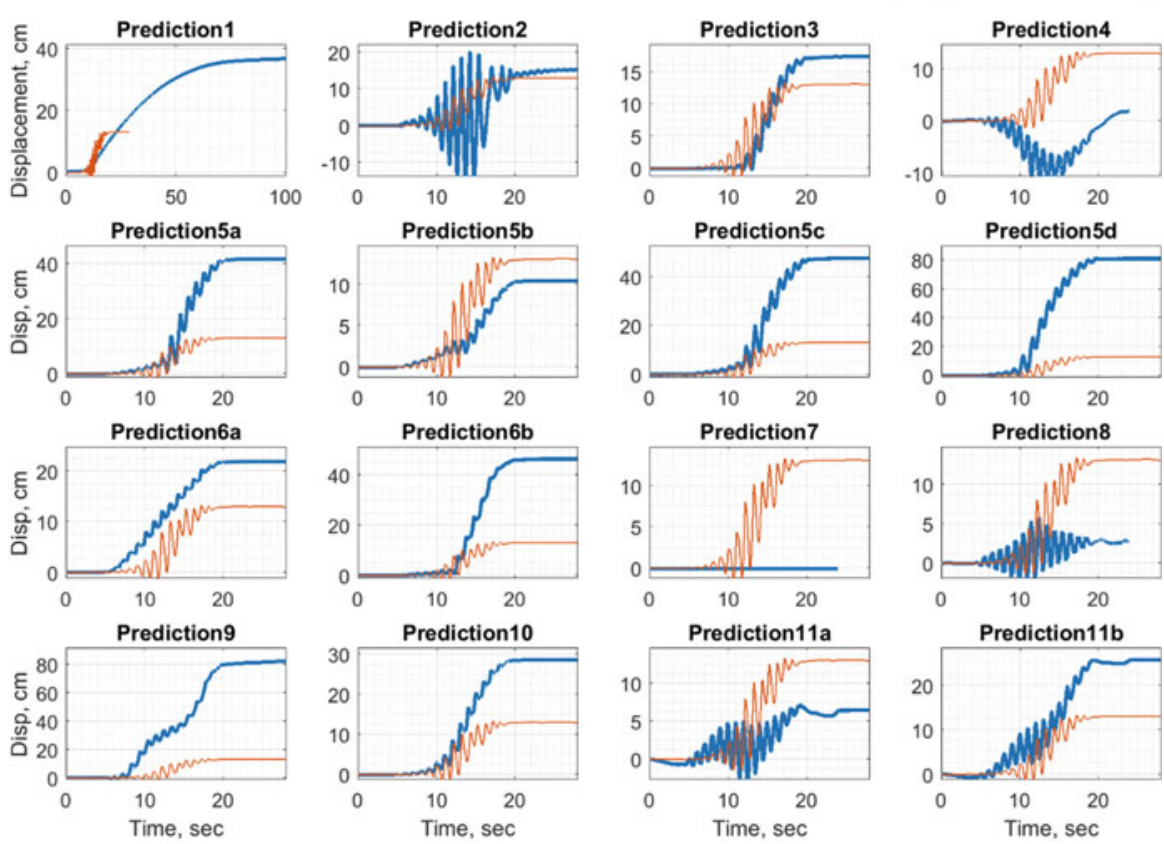

Fig. 10.25 Comparisons of the numerical simulations of lateral displacement with the measured time history of surface displacement in the UCD-3 test
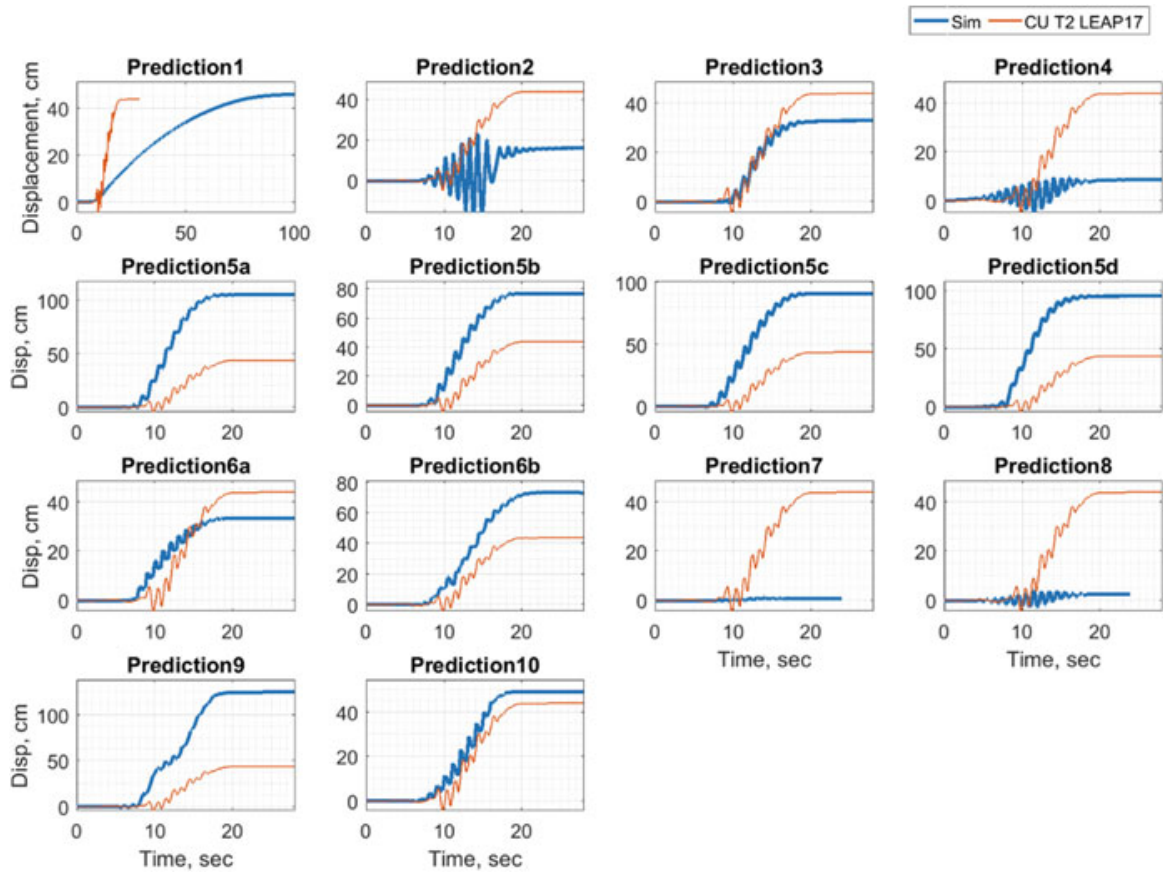

Fig. 10.26 Comparisons of the numerical simulation of lateral displacement with the constructed time history of the surface lateral displacement in the CU-2 test 

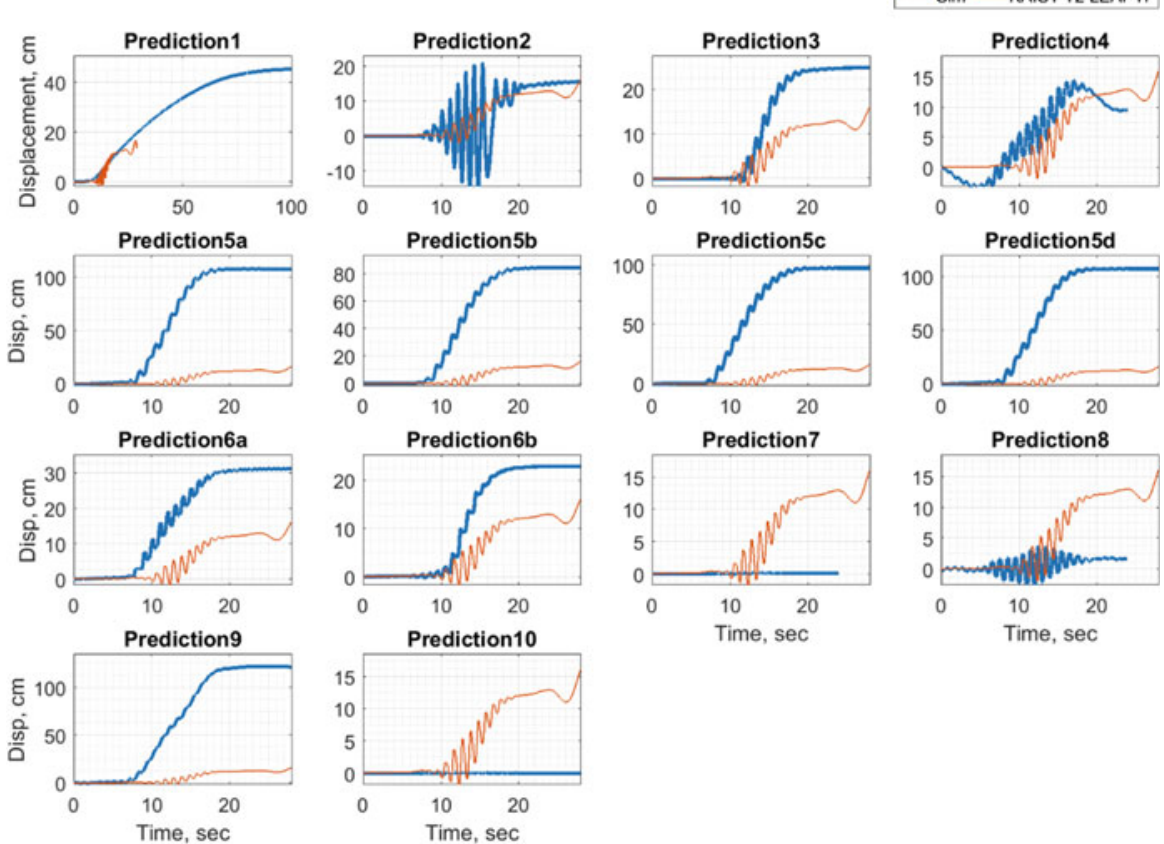

Time, sec

Fig. 10.28 Comparisons of the numerical simulation of lateral displacement with the constructed time history of the surface lateral displacement in the KAIST-2 test
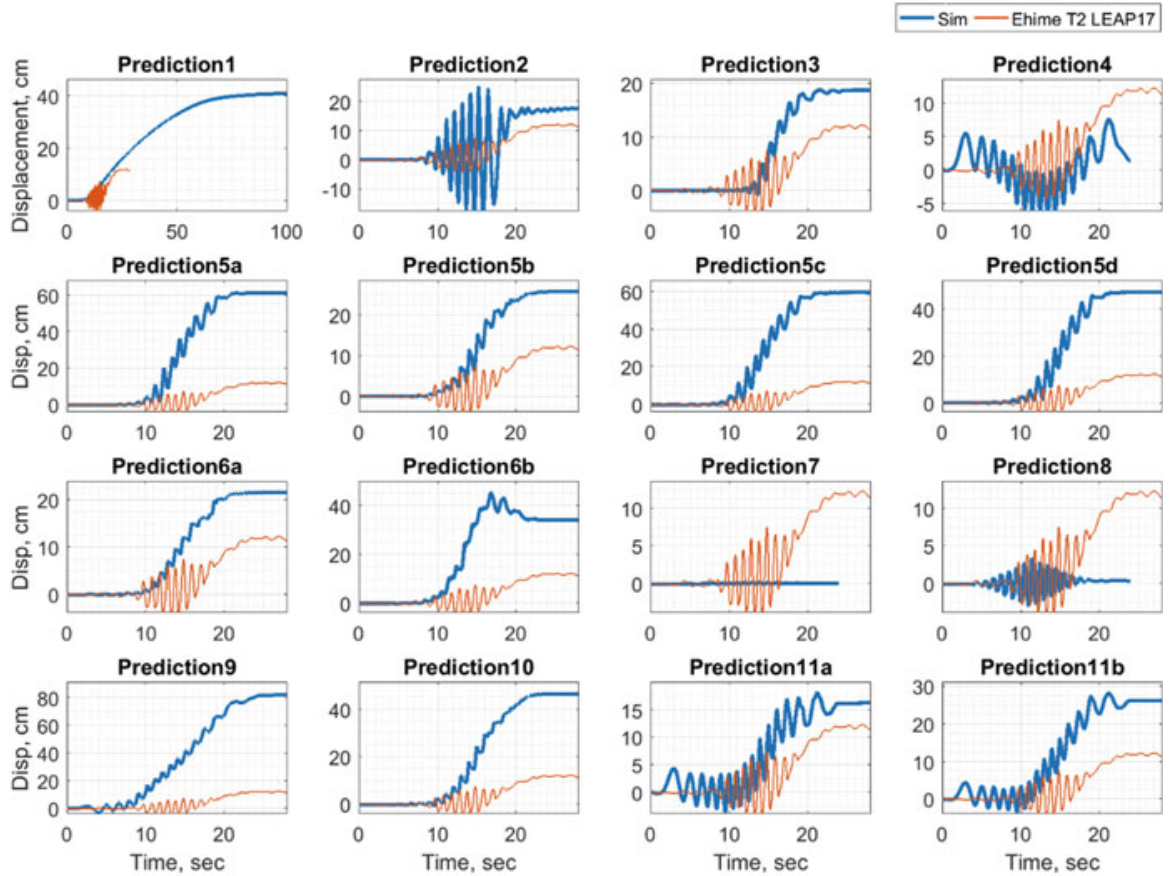

Fig. 10.27 Comparisons of the numerical simulation of lateral displacement with the constructed time history of the surface lateral displacement in the Ehime-2 tests 

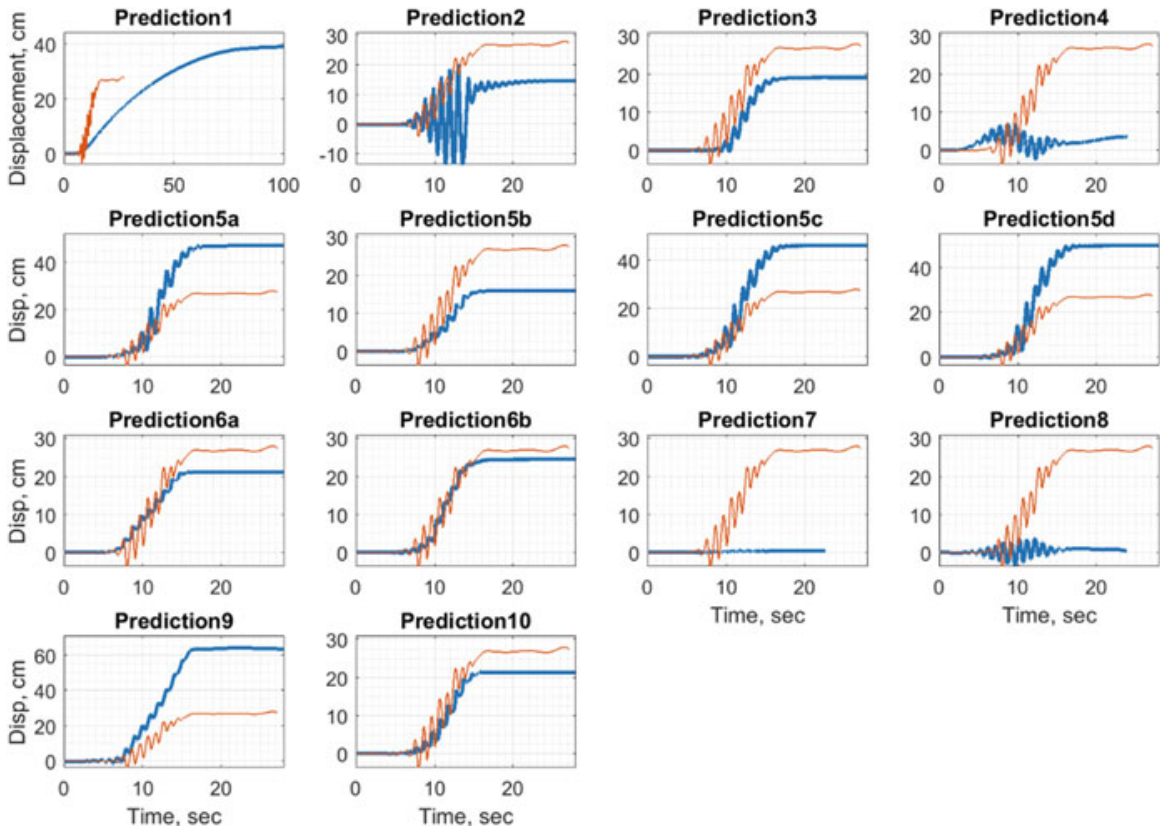

Fig. 10.29 Comparisons of the numerical simulation of lateral displacement with the constructed time history of the surface lateral displacement in NCU-3 test
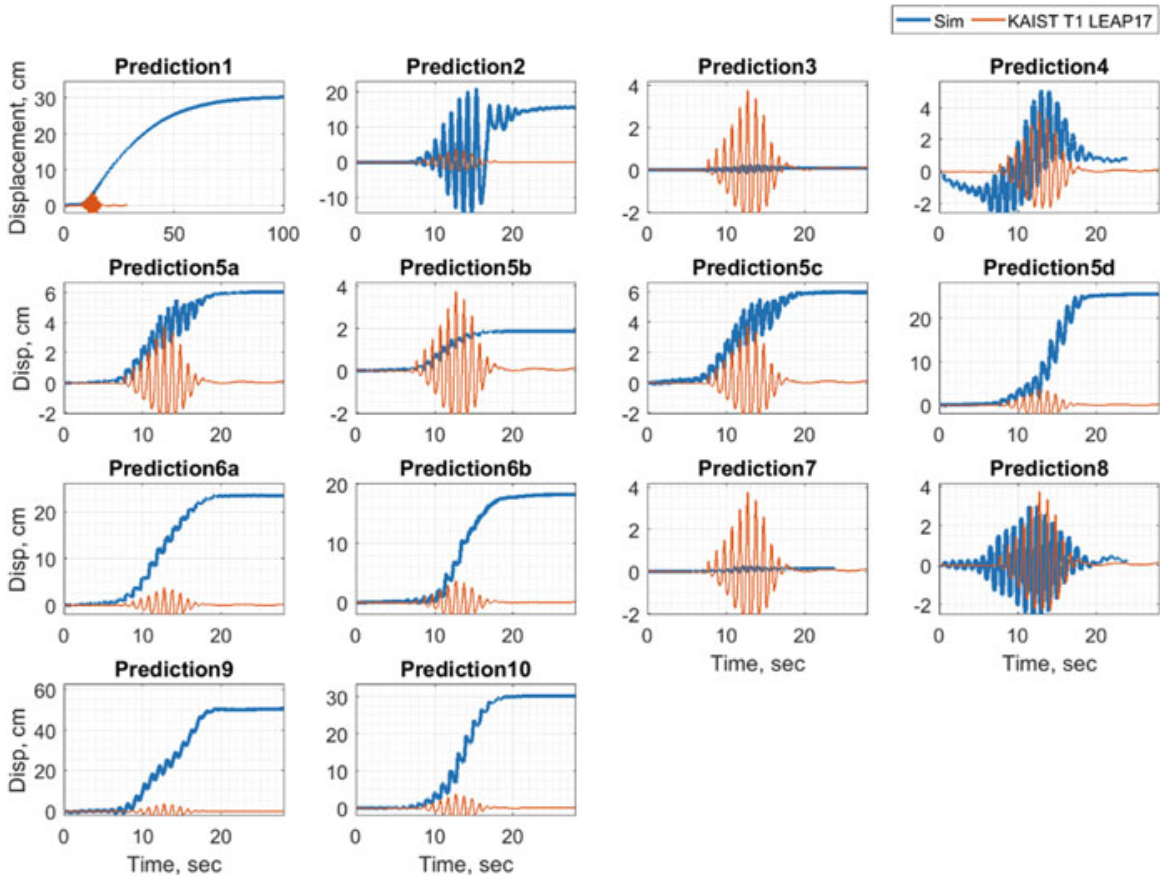

Fig. 10.30 Comparisons of the numerical simulations of lateral displacement with the time history of dynamic components of lateral displacement in the KAIST-1 test 

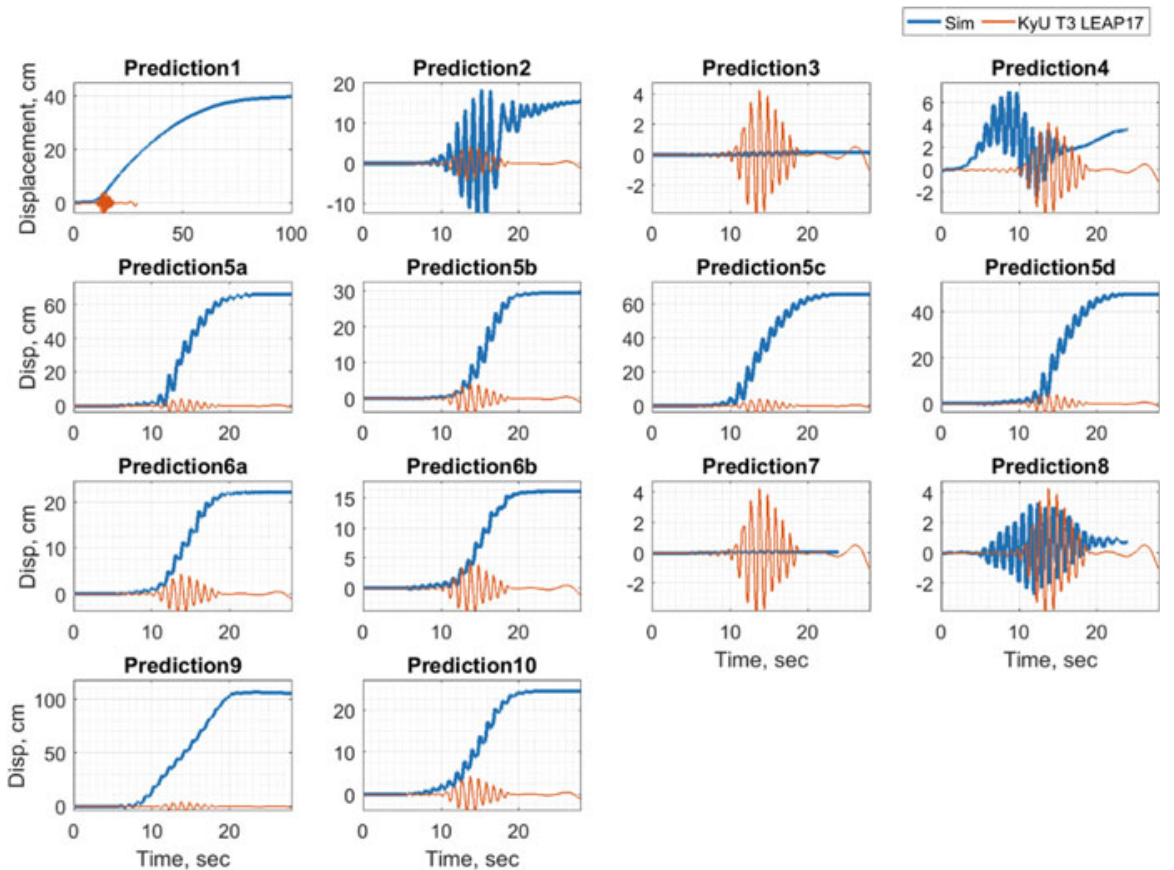

Fig. 10.31 Comparisons of the numerical simulations of lateral displacement with the time history of dynamic components of lateral displacement in the KyU-3 tests

- The majority of Type-B simulations predicted much larger displacements than those observed in UCD-1 test. Liquefaction was not triggered in the experiment, but apparently it was triggered in many of the simulations. The final displacements (but not the cyclic components of displacement) predicted by Predictions 4,7 , and 11a are comparable with the very small displacement reported for the UCD-1 test.

- A number of predictions (i.e., 2, 3, 5b, 6a) showed maximum lateral displacements that favorably compare with the observed responses in the UCD-3 test. A large number of other simulations also showed comparable trends but overpredicted the lateral displacements. It is also noted that a number of simulations were able to predict the magnitude of the cyclic component of the lateral displacements quite well $(4,5 \mathrm{a}, 5 \mathrm{c}, 8,11 \mathrm{~b})$. A few other predictions $(1,3,6 \mathrm{a}, 10)$ underpredicted the cyclic component of the lateral displacement and showed a more or less smooth rise to the final displacement. This particular aspect of the simulation results is likely related to the cyclic stress-strain responses produced by the different constitutive models. Few models show small opening of the stress-strain loops and exhibit significant ratcheting, while a few others display increasingly larger stress-strain loops without much ratcheting (see Manzari et al. 2019).

- An interesting trend is observed in the simulations of the CU-2 test which produced the largest observed lateral displacement consistent with the lowest 
value of cone penetration resistance measured in this test. Prediction 10 was closest to the "experimental" results. A number of numerical simulations overpredicted the lateral displacement in this test $(5 a, 5 b, 5 c, 6 b, 9)$ while others significantly underpredicted $(1,2,4,7,8)$ the lateral displacement. It must be noted, however, that the constitutive models used in the numerical simulations were calibrated for densities higher than $65 \%$ and the relatively low density achieved in CU-2 was outside of the range of densities the models were calibrated for.

- Significant underpredictions are observed in all the displacement simulations provided by prediction team 7 . Based on the comments provided by the prediction team 7 in the LEAP-2017 workshop at UC Davis, these numerical simulations were based on a set of model parameters that were erroneously selected. This error has been corrected later and the corrected results are presented and discussed in the paper by Wada and Ueda (2019).

- Prediction 1 shows very little cyclic displacements (overly smooth curves) and a very long delayed rise of lateral displacements after the end of base motion. Interestingly, the ultimate displacement was approximately $40 \mathrm{~cm}$ for every simulation. This is not consistent with the observed experimental trends and the simulations provided by other predictors. The reason for this discrepancy has been discussed in Wang et al. (2019).

- Overall, the maximum lateral displacements predicted by prediction teams 2 and 3 appeared to be closer to the "measured" displacements. However, Prediction 2 displayed much larger cyclic displacements than those observed in the experiments and Prediction 3 tended to underpredict the cyclic components of displacement.

\subsection{Overall Performance of Numerical Simulations}

To further assess the overall performance of the predictions and the quality of their fit to the centrifuge test results, the following indicators are selected: (1) the maximum lateral displacement at the center of the soil surface, (2) the maximum excess pore water pressure ratio achieved at the depth of $1 \mathrm{~m}(\mathrm{P} 4)$, and (3) a scalar representing a measure of spectral acceleration (MSA). The root mean square error (RMSE) of these indicators was computed for each numerical team based on the nine centrifuge experiments as:

$$
\mathrm{RMSE}=\sqrt{\frac{1}{N} \sum_{N}\left(P^{e}-P^{s}\right)^{2}}
$$

where $P^{e}$ and $P^{s}$ are the values of an indicator for the experiments and simulation, respectively, and $N$ is the number of experiments which is equal to 9 for all the 
predictions except for the predictions $11 \mathrm{a}$ and $11 \mathrm{~b}$ which were reported for only three centrifuge experiments.

The following measures are used to represent the spectral accelerations:

$$
M S a_{1}=\frac{(S a)_{1 \mathrm{~Hz}}+(S a)_{3 \mathrm{~Hz}}+(S a)_{20 \mathrm{~Hz}}}{3} ; M S a_{2}=\int_{0.5}^{20} S_{a} d f
$$

$M S a_{1}$ is the average of spectral values at $1 \mathrm{~Hz}, 3 \mathrm{~Hz}$, and $20 \mathrm{~Hz}$ that represents the peak of each acceleration time history, while $M S a_{2}$ is essentially the area under the spectral acceleration graph ( $\mathrm{Sa}$ versus $f$ ).

Figures 10.32 and 10.33 summarize the results of the computed RMSEs for the lateral displacement on the ground surface $(d x)$, the maximum excess pore pressure ratio at the location of $\mathrm{P} 4\left(r_{\mathrm{u}}-\mathrm{P} 4\right)$, and the spectral acceleration at the location of AH4 (MSa-AH4). Figure 10.32 presents the RMSEs computed based on $M S a_{1}$ and Figure 10.33 presents the RMSE computed based on $M S a_{2}$. These figures present a sample of the overall fit of the predictions to the experimental data. The RMSEs of $\mathrm{MSa}_{1}$ and $\mathrm{MSa}_{2}$ for Prediction 2 exceed the limits of the plot. This is mainly due to the presence of large high-frequency components in the acceleration time history as shown in Figs. 10.10, 10.11, 10.12, 10.13, 10.14, 10.15, and 10.16.

It is observed that Predictions 2, 3, 6a, and 11a show reasonably small RMSEs for lateral displacements, while Predictions 1, 2, 3, 5a, 5d, 6a, 8, and 9 show relatively small RMSEs for excess pore pressure ratios, $r_{\mathrm{u}}$, at $\mathrm{P}_{4}$. The spectral accelerations predicted by team 11 (11b and 11a) at AH4 show the lowest RMSEs.

The RMSEs are recomputed for the three centrifuge tests that were predicted by numerical simulation team 11 in order to assess the effect of number of centrifuge tests used. Figure 10.34 shows the results of these recalculated RMSEs for lateral displacements, pore pressure ratios at $\mathrm{P} 4$, and spectral accelerations $\left(\mathrm{MSa}_{2}\right)$ at $\mathrm{AH} 4$. While there are some changes (e.g., significant reduction in RMSE of lateral displacements predicted by teams 1 and $5 \mathrm{~b}$ ) in the relative performance of individual predictions, the overall trend observed in Fig. 10.33 remains the same. Hence, it was decided that it is not unreasonable to include the predictions made by team 11 in the comparisons with other predictions. In the following figures, the approach used in Fig. 10.33 will be used (i.e., $N=9$ for all predictions, except for team 11 where $N=3$ ).

Figures 10.35, 10.36, and 10.37 show similar comparisons as those shown in Figs. 10.32 and 10.33 for excess pore water pressure at locations of P3, P2, and P1 and for spectral accelerations $\mathrm{AH} 3, \mathrm{AH} 2$, and $\mathrm{AH} 1$, respectively. It is interesting to note that differences between the results of experiments and numerical simulations steadily increase as the point of interest moves from the bottom of the soil deposit to the ground surface. 


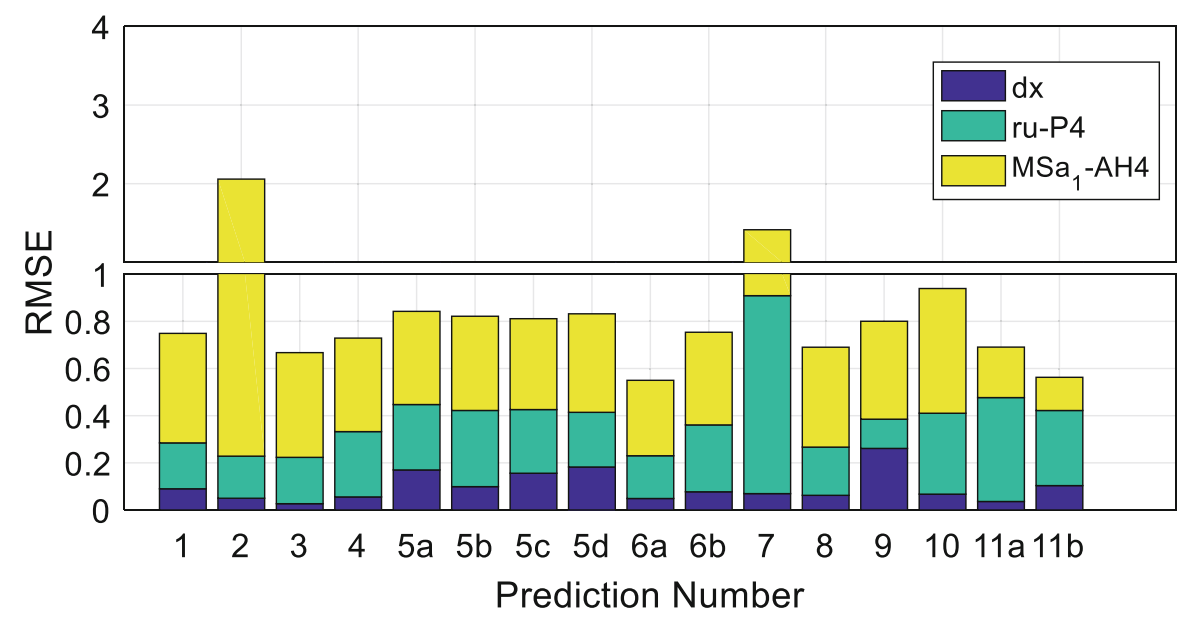

Fig. 10.32 RMSE values for the Type-B simulations compared to the observed lateral displacements, pore pressure ratios at $\mathrm{P} 4$, and spectral accelerations $\left(M S a_{1}\right)$ at $\mathrm{AH} 4$

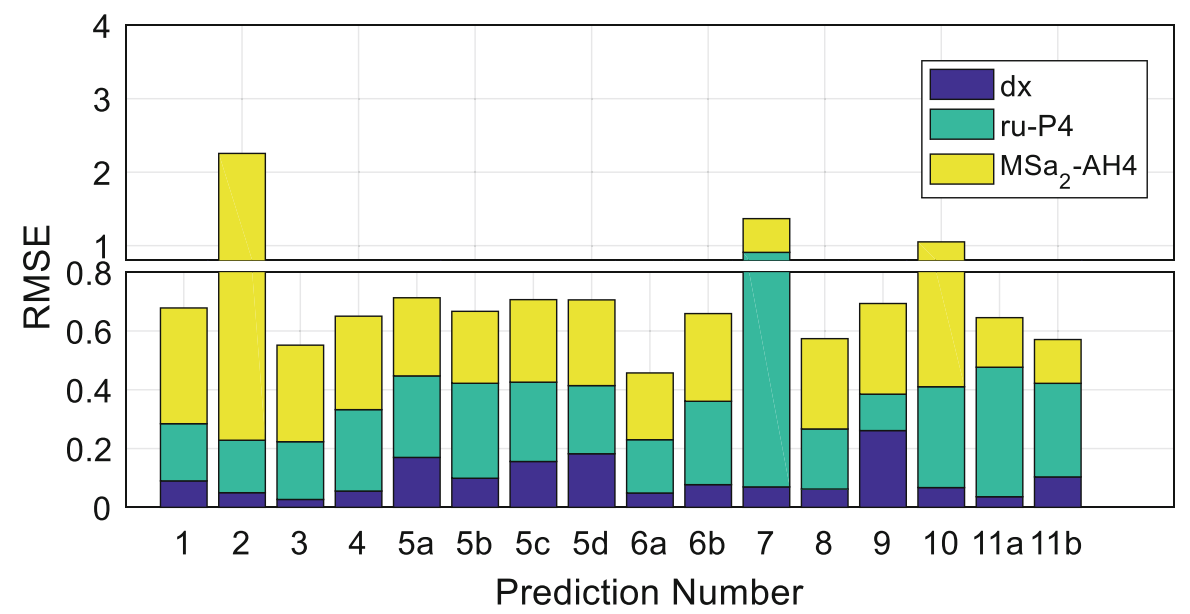

Fig. 10.33 RMSE values for the Type-B simulations compared to the observed lateral displacements, pore pressure ratios at $\mathrm{P} 4$, and spectral accelerations $\left(M \mathrm{Ma}_{2}\right)$ at $\mathrm{AH} 4$ 


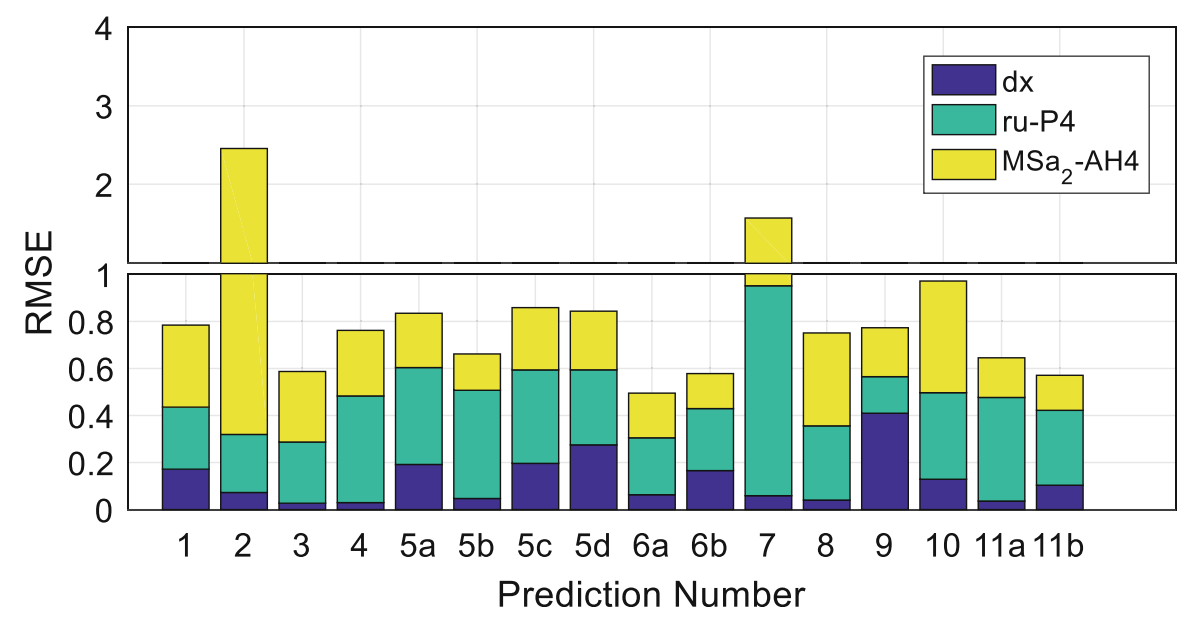

Fig. 10.34 Recomputed RMSE values considering only the three centrifuge test that were predicted by the numerical simulation team 11

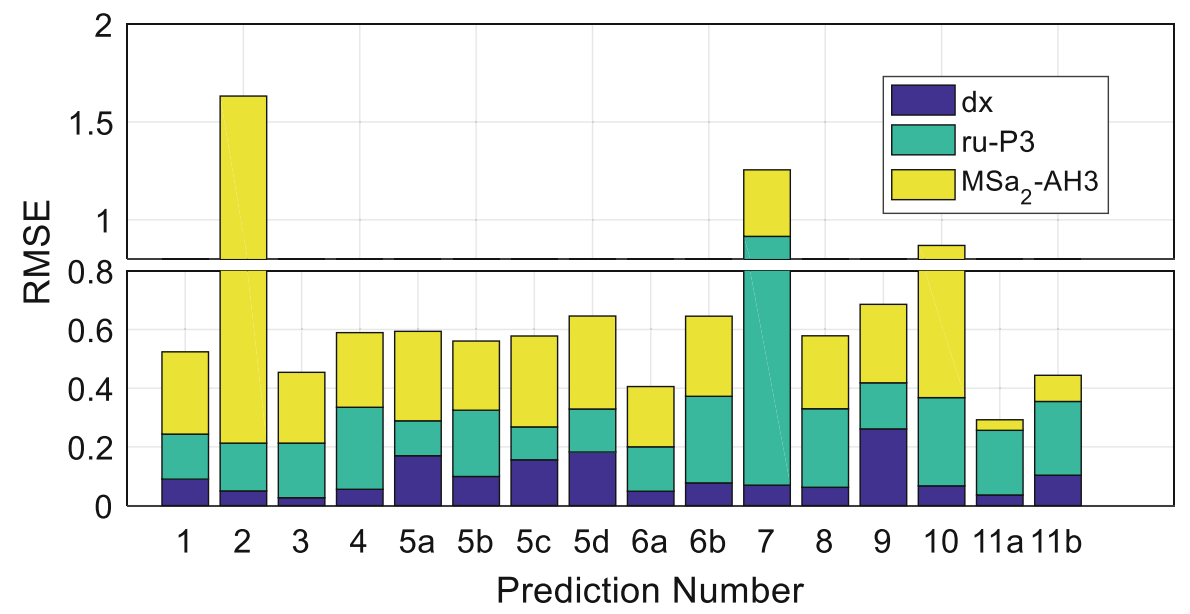

Fig. 10.35 RMSE values for the Type-B simulations compared to the observed lateral displacements, pore pressure ratios at $\mathrm{P} 3$, and spectral accelerations $\left(\mathrm{MSa}_{2}\right)$ at $\mathrm{AH} 3$ 


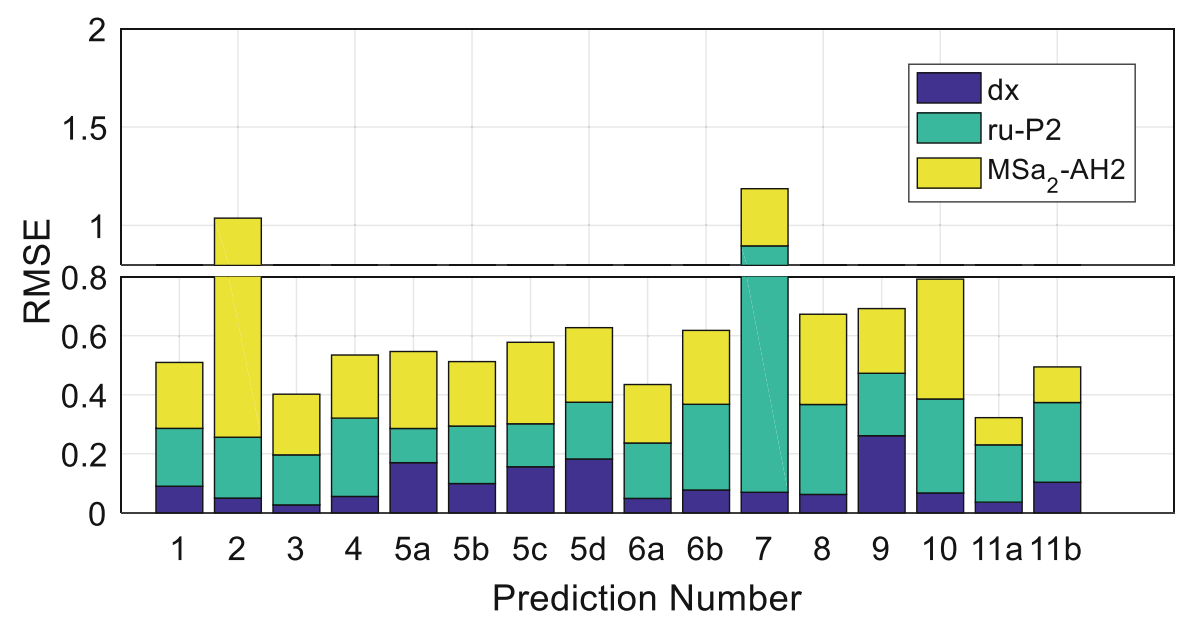

Fig. 10.36 RMSE values for the Type-B simulations compared to the observed lateral displacements, pore pressure ratios at $\mathrm{P} 2$, and spectral accelerations $\left(\mathrm{MSa}_{2}\right)$ at $\mathrm{AH} 2$

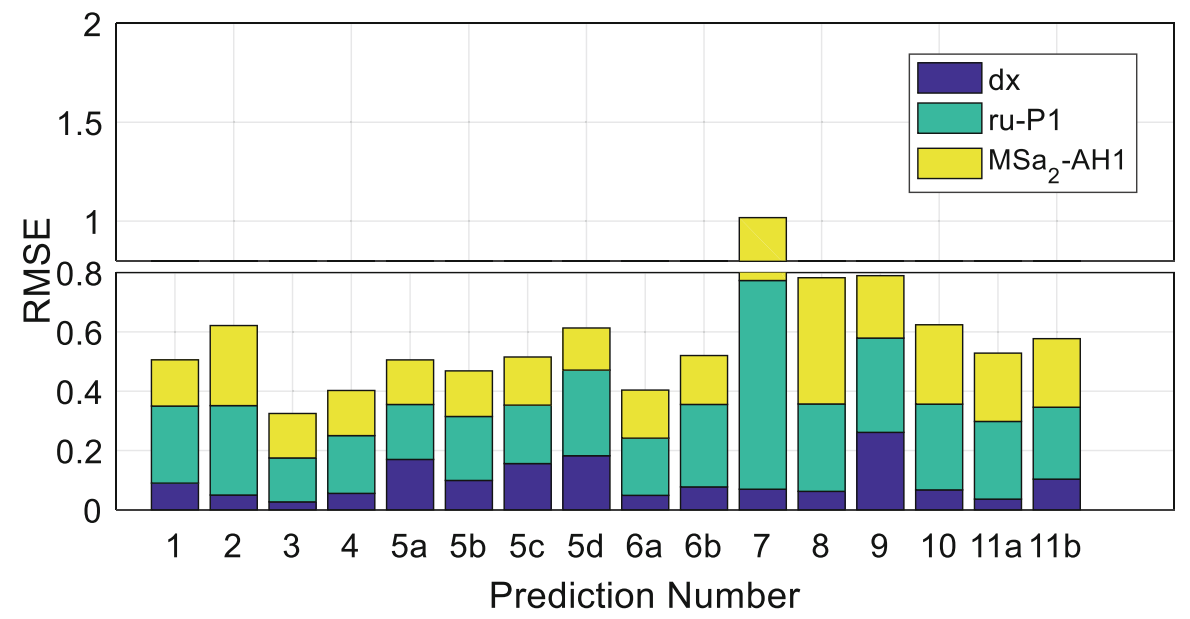

Fig. 10.37 RMSE values for the Type-B simulations compared to the observed lateral displacements, pore pressure ratios at $\mathrm{P} 1$, and spectral accelerations $\left(\mathrm{MSa}_{2}\right)$ at $\mathrm{AH} 1$

\subsection{Conclusions}

Many of the Type-B simulations submitted by the participating prediction teams show trends that are comparable to the measured results obtained for the selected LEAP-2017 centrifuge tests. While the differences between the simulations and experiments are smaller for a few predictions, predicting all the key quantities (excess pore pressure, displacement, cyclic displacement, and acceleration) for the selected tests remains a challenging task that can be potentially addressed by reassessing the performance of the participating numerical simulation platforms in 
simulation of all the centrifuge data produced in LEAP-2017 project. Moreover, additional centrifuge tests with detailed measurements of displacement time histories can provide a more solid basis for future comparisons of the numerical simulations and experiments in geotechnical problems where permanent displacements are of significant interest.

Acknowledgments The experimental work and numerical simulations on LEAP-UCD-2017 were supported by different funds depending mainly on the location of the work. The work by the US PIs (Manzari, Kutter, and Zeghal) is funded by National Science Foundation grants: CMMI 1635524, CMMI 1635307, and CMMI 1635040. Partial funding from Caltrans supported the efforts of co-authors Elgamal and Qiu.

\section{References}

Carey, T. J., Stone, N., Hajialilue Bonab, M., \& Kutter, B. L. (2019). LEAP-UCD-2017 centrifuge test at University of California, Davis. In B. Kutter et al. (Eds.), Model tests and numerical simulations of liquefaction and lateral spreading: LEAP-UCD-2017. New York: Springer.

Chen, L., Ghofrani, A., \& Arduino, P. (2019). Prediction of LEAP-UCD-2017 centrifuge test results using two advanced plasticity sand models. In B. Kutter et al. (Eds.), Model tests and numerical simulations of liquefaction and lateral spreading: LEAP-UCD-2017. New York: Springer.

El Ghoraiby, M. A., Park, H., \& Manzari, M. (2018). LEAP-2017 GWU laboratory tests. DesignSafe-CI, Dataset. https://doi.org/10.17603/DS2210X.

El Ghoraiby, M. A., Park, H., \& Manzari, M. T. (2019). Physical and mechanical properties of Ottawa F65 Sand. In B. Kutter et al. (Eds.), Model tests and numerical simulations of liquefaction and lateral spreading: LEAP-UCD-2017. New York: Springer.

Fasano, G., Chiaradonna, A., \& Bilotta, E. (2019). LEAP-UCD-2017 centrifuge test simulation at UNINA. In B. Kutter et al. (Eds.), Model tests and numerical simulations of liquefaction and lateral spreading: LEAP-UCD-2017. New York: Springer.

Fukutake, K., \& Kiriyama, T. (2019). LEAP-2017 centrifuge test simulation using HiPER. In B. Kutter et al. (Eds.), Model tests and numerical simulations of liquefaction and lateral spreading: LEAP-UCD-2017. New York: Springer.

Hung, W.-Y., \& Liao, T.-W. (2019). LEAP-UCD-2017 centrifuge tests at NCU. In B. Kutter et al. (Eds.), Model tests and numerical simulations of liquefaction and lateral spreading: LEAPUCD-2017. New York: Springer.

Ichii, J., Uemura, K., Orai, N., \& Hyodo, J. (2019). Numerical simulation trial by cocktail glass model in FLIP ROSE for LEAP-UCD-2017. In B. Kutter et al. (Eds.), Model tests and numerical simulations of liquefaction and lateral spreading: LEAP-UCD-2017. New York: Springer.

Kim, S.-N., Ha, J.-G., Lee, M.-G., \& Kim, D.-S. (2019). LEAP-UCD-2017 centrifuge test at KAIST. In B. Kutter et al. (Eds.), Model tests and numerical simulations of liquefaction and lateral spreading: LEAP-UCD-2017. New York: Springer.

Kutter, B., Carey, T., Hashimoto, T., Zeghal, M., Abdoun, T., Kokalli, P., Madabhushi, G., Haigh, S., Hung, W.-Y., Lee, C.-J., Iai, S., Tobita, T., Zhou, Y. G., Chen, Y., \& Manzari, M. T. (2017). LEAP-GWU-2015 experiment specifications, results, and comparisons. International Journal of Soil Dynamics and Earthquake Engineering, 113, 616. https://doi.org/10.1016/j.soildyn. 2017.05.018.

Kutter, B. L., Carey, T. J., Bonab, M. H., Stone, N., Manzari, M., Zeghal, M., Escoffier, S., Haigh, S. K., Madabhushi, G., Hung, W., Kim, D., Kim, N., Okamura, M., Tobita, T., Ueda, K., \& Zhou, Y. (2019). LEAP-UCD 2017 version 1.01 model specifications. In B. Kutter et al. (Eds.), Model tests and numerical simulations of liquefaction and lateral spreading: LEAP-UCD-2017. New York: Springer. 
Liu, K., Zhou, Y.-G., She, Y., Xia, P., Meng, D., Huang, J.-S., Yao, G., \& Chen, Y.-M. (2019). Specifications and results of centrifuge model test at Zhejiang University for LEAP-UCD-2017. In B. Kutter et al. (Eds.), Model tests and numerical simulations of liquefaction and lateral spreading: LEAP-UCD-2017. New York: Springer.

Madabhushi, S. S. C., Dobrisan, A., Beber, R., Haigh, S. K., \& Madabhushi, S. P. G. (2019). LEAPUCD-2017 centrifuge tests at Cambridge. In B. Kutter et al. (Eds.), Model tests and numerical simulations of liquefaction and lateral spreading: LEAP-UCD-2017. New York: Springer.

Manzari, M., Ghoraiby, M. E., Kutter, B. L., Zeghal, M., Abdoun, T., Arduino, P., Armstrong, R. J., Beaty, M., Carey, T., Chen, Y.-M., Ghofrani, A., Gutierrez, D., Goswami, M., Haigh, S. K., Hung, W.-Y., Iai, S., Kokkali, P., Lee, C.-J., Madabhushi, S. P. G., Mejia, L., Sharp, M., Tobita, T., Ueda, K., Zhou, Y.-G., \& Ziotopoulou, K. (2017). Liquefaction analysis and experiment projects (LEAP): Summary of observations from the planning phase. International Journal of Soil Dynamics and Earthquake Engineering, 113, 714. https://doi.org/10.1016/j.soildyn.2017.05.015.

Manzari, M. T., El Ghoraiby, M. A., Kutter, B. L., Zeghal, M., Wang, R., Chen, R., Zhang, J.-M., Osamu Ozutsumi, O., Fukutake, K., Kiriyama, T., Fasano, G., Chiaradonna, A., Bilotta, E., Montgomery, J., Ziotopoulou, K., Chen, L., Ghofrani, A., Arduino, P., Wada, T., Ueda, K., Mercado, V., Fuentes, W., Lascarro, C., Yang, M., Barrero, A. R., Taiebat, M., Tsiaousi, D., Ugalde, J., Thaleia Travasarou, T., Ichii, K., Uemura, K., Orai, N., Hyodo, M., Abdoun, T., Haigh, S., Madabhushi, S. P. G., Tobia, T., Hung, W.-Y., Kim, D. S., Okamura, M., Zhou, Y.G., \& Escoffier, S. (2019). LEAP-2017 simulation exercise: Calibration of constitutive models and simulation of the element test. In B. Kutter et al. (Eds.), Model tests and numerical simulations of liquefaction and lateral spreading: LEAP-UCD-2017. New York: Springer.

Mercado, V., Fuentes, W., \& Lascarro, C. (2017). LEAP 2017 simulation exercise - Phase I: Model calibration. Calibration report, June 28, DesignSafe.

Montgomery, J., \& Ziotopoulou, K. (2019). Numerical simulations of selected LEAP centrifuge. In B. Kutter et al. (Eds.), Model tests and numerical simulations of liquefaction and lateral spreading: LEAP-UCD-2017. New York: Springer.

Okamura, M., \& Nurani Sjafruddin, A. (2019). LEAP-2017 centrifuge test at Ehime University. In B. Kutter et al. (Eds.), Model tests and numerical simulations of liquefaction and lateral spreading: LEAP-UCD-2017. New York: Springer.

Ozutsumi, O. (2019). LEAP-UCD-2017 numerical simulation at Meisosha Corp. In B. Kutter et al. (Eds.), Model tests and numerical simulations of liquefaction and lateral spreading: LEAP$U C D$-2017. New York: Springer.

Tsiaousi, D., Ugalde, J., \& Travasarou, T. (2019). LEAP-UCD-2017 simulation team Fugro. In B. Kutter et al. (Eds.), Model tests and numerical simulations of liquefaction and lateral spreading: LEAP-UCD-2017. New York: Springer.

Vargas Tapia, R. R., Tobita, T., Ueda, K., \& Yatsugi, H. (2019). LEAP-UCD-2017 Centrifuge test at Kyoto University. In B. Kutter et al. (Eds.), Model tests and numerical simulations of liquefaction and lateral spreading: LEAP-UCD-2017. New York: Springer.

Wada, T., \& Ueda, K. (2019). LEAP-UCD-2017 type-B predictions through FLIP at Kyoto University. In B. Kutter et al. (Eds.), Model tests and numerical simulations of liquefaction and lateral spreading: LEAP-UCD-2017. New York: Springer.

Wang, R., Chen, R., \& Zhang, J.-M. (2019). LEAP-UCD-2017 simulations at Tsinghua University. In B. Kutter et al. (Eds.), Model tests and numerical simulations of liquefaction and lateral spreading: LEAP-UCD-2017. New York: Springer.

Yang, M., Barrero, A. R., \& Taiebat, M. (2019). Application of a SANISAND model for numerical simulations of the LEAP 2017 experiments. In B. Kutter et al. (Eds.), Model tests and numerical simulations of liquefaction and lateral spreading: LEAP-UCD-2017. New York: Springer.

Zeghal, M., Goswami, N., Kutter, B., Manzari, M. T., Abdoun, T., Arduino, P., Armstrong, R., Beaty, M., Chen, Y.-M., Ghofrani, A., Haigh, S., Hung, Y.-W., Iai, S., Kokkali, P., Lee, C.-J., Madabhushi, G., Tobita, T., Ueda, K., Zhou, Y.-G., \& Ziotopoulou, K. (2017). Stress-strain response of the LEAP-2015 centrifuge tests and numerical predictions. International Journal of Soil Dynamics and Earthquake Engineering., 113, 804. https://doi.org/10.1016/j.soildyn.2017. 10.014 . 
Open Access This chapter is licensed under the terms of the Creative Commons Attribution 4.0 International License (http://creativecommons.org/licenses/by/4.0/), which permits use, sharing, adaptation, distribution and reproduction in any medium or format, as long as you give appropriate credit to the original author(s) and the source, provide a link to the Creative Commons license and indicate if changes were made.

The images or other third party material in this chapter are included in the chapter's Creative Commons license, unless indicated otherwise in a credit line to the material. If material is not included in the chapter's Creative Commons license and your intended use is not permitted by statutory regulation or exceeds the permitted use, you will need to obtain permission directly from the copyright holder. 